\title{
Green Technology and Patents in the Presence of Green Consumers*
}

\author{
Corinne Langinier ${ }^{\dagger}$ and Amrita Ray Chaudhuri ${ }^{\ddagger}$
}

May 2019

\begin{abstract}
We develop a theoretical framework to investigate the impact of patent policies and emission taxes on green innovation that reduces the emission output ratio, and on the emission level. In the absence of green consumers, the introduction of patents results in a paradox whereby increasing emission tax beyond a certain threshold leads to a discrete increase in the emission level, which may be avoided by reducing the patenting cost. In the presence of green consumers, this paradox is restricted to an intermediate range of tax rates, and at sufficiently high tax rates, reducing the patenting cost may increase the emission level. Also, higher emission taxes increase green investment only if the fraction of green consumers is sufficiently small, and the magnitude of this effect decreases as this fraction increases. Moreover, a stricter patentability requirement is only effective at reducing emissions if the fraction of green consumers is sufficiently small.
\end{abstract}

Keywords: Patent, Clean Technologies, Environmentally Friendly Consumers JEL Classification $=$ O34, L13, Q50

${ }^{*}$ This work was carried out with the financial support of SAS grant, University of Alberta. We are grateful to Agamani Chakrabarty for her research assistance. We would like to thank Stefan Ambec, Sophie Bernard, Philippe Marcoul, and the participants at the seminar at Queen's University, Smith School of Business, REES department, University of Alberta, TECHNIS webinar, University of Crete, and of CAES 2017, IIOC 2018, CEA 2018 and WCERE 2018 for insightful comments. All remaining errors are ours.

${ }^{\dagger}$ Department of Economics, University of Alberta, Edmonton, Canada (langinie@ualberta.ca)

${ }^{\ddagger}$ Department of Economics, University of Winnipeg, Canada, CentER \& TILEC (a.raychaudhuri@uwinnipeg.ca) 


\section{Introduction}

Given increasing environmental concerns and the increasing environmental consciousness of citizens globally, developing green technologies has become a key policy initiative of international organizations such as the UN and the G8, and of national governments. ${ }^{1}$ Within this context, we examine the interaction of patent policies and environmental regulation in enhancing innovation in "green", or less polluting, production technologies, and in reducing the emission of pollutants. Studying patent policies for the development of green technologies requires a specific analysis due to potential interactions between knowledge and environmental externalities caused by the innovators. We thus develop a theoretical framework to investigate the impact of changing patentability requirements and patenting costs in conjunction with increasing emission taxes, allowing for the presence of environmentally friendly consumers.

This paper builds upon existing results in the literatures on the development of green technologies, on patents, and on the environmentally friendly behaviour of consumers.

The literature on green technologies is rapidly evolving in response to global environmental problems such as climate change. Climate experts propose that the increase in average global temperature should be restricted to about $2^{\circ} \mathrm{C}$ to avoid the possibility of catastrophic damage, a goal that may only be reached by developing and implementing "breakthrough" technologies that reduce emissions dramatically (Barrett, 2009; Galiana and Green, 2009). One stream of the literature has evolved around the seminal work of Porter (1991) and Porter and van der Linde (1995), referred to as the Porter Hypothesis, and examines whether the implementation of stricter environmental regulations increases firms' incentives to invest in green Research and Development $(\mathrm{R} \& \mathrm{D})$. The empirical evidence surrounding the Porter Hypothesis is mixed (Ambec et al., 2013). Our paper is more closely related to a second stream of the literature, which argues

\footnotetext{
${ }^{1}$ In 2016, the Canadian federal government announced that it will invest $\$ 200$ million annually to create sector specific strategies to support the development of clean technologies and invest $\$ 100$ million annually in organizations that support clean technology firms such as Sustainable Development Technology Canada. In the U.S., the Department of Energy's Loan Program Office has more than $\$ 40$ billion in remaining loans to help finance innovative technologies that can reduce carbon emissions. In Canada and the U.S. respectively, about 2500 and 18500 patents for green technologies are issued annually.
} 
that given the combination of environmental externalities and knowledge market failures facing regulators, environmental policies are not sufficient to achieve the first best social outcome, and need to be combined with policies addressing the relevant knowledge market failure (Carraro and Siniscalco, 1994; Carraro and Soubeyran, 1996; Katsoulacos and Xepapadeas, 1996; Fischer and Newell, 2008; Gerlagh et al., 2009; Acemoglu et al., 2012; Hepburn et al., 2018; Lehmann and Söderholm, 2018; Popp, 2019).

Examining patent systems within this context is important due to the lack of consensus among policy-makers and academics regarding the role of patents in promoting $\mathrm{R} \& \mathrm{D}$ in general and green technologies in particular. ${ }^{2}$ On the one hand, international organizations advocate royalty-free compulsory licensing of green technologies, excluding green technologies from patenting, and even revoking existing patent rights on them (UNFCCC, 2009). ${ }^{3}$ On the other hand, many countries actively lower the cost of obtaining patents for green innovations.

Our focus is on the role played by two different aspects of patent policies, patenting costs and patentability requirements, and how they interact with emission taxes in fostering green innovation. ${ }^{4}$ The cost associated with obtaining and implementing patents can be significant and comprises a number of components. First, the monetary fees associated with the application process range on average between $\$ 5000-\$ 15000$. Second, there exists an opportunity cost in terms of lost profits while waiting for the patent to be granted. On average, the waiting time is about three years and is frequently longer. ${ }^{5}$ Third, the potential litigation costs of enforcing a patent may be large enough to deter small firms from obtaining patents in several industries. ${ }^{6}$

\footnotetext{
${ }^{2}$ Patents address the problem due to the externality that results from imperfect appropriability of knowledge by endowing innovators with property rights on their inventions. A patent confers its owner a temporary right to exclude others from exploiting the innovation. In exchange for the exclusionary right, the patent holder must disclose his innovation. For surveys of the patent literature, see Langinier and Moschini (2002), Rockett (2010), Eckert and Langinier (2014).

${ }^{3}$ Such provisions are also incorporated in the Agreement on Trade Related Aspects of Intellectual Property Rights (TRIPS) (Derclaye, 2008; Rimmer, 2011).

${ }^{4}$ Gerlagh et al., (2014) is another paper to examine patent policies for green technologies. It focuses on analyzing the impact of changing the lifetime of patents issued to green innovations.

${ }^{5}$ See Eckert and Langinier (2014).

${ }^{6}$ According to the American Intellectual Property Law Association, the cost of an average patent lawsuit, where $\$ 1$ million to $\$ 25$ million is at risk, is $\$ 1.6$ million through the end of discovery and $\$ 2.8$ million through
} 
Policy makers use different means to reduce these patenting costs. One such method which has been frequently used is fast-tracking, or expediting the review process, of green patent applications. This is a key policy initiative undertaken by several countries including Australia, Brazil, Canada, China, UK, U.S., Japan and Korea. Given that the time from application to grant has been effectively reduced by up to $75 \%$ for patents entering the fast track procedure and that evidence shows that fast-tracking programs have accelerated the diffusion of knowledge in green technologies in the short run (Dechezleprêtre, 2013), such policies are likely to continue and proliferate. In our model, we introduce a lump sum cost associated with obtaining and implementing patents, and examine the impact of lowering this cost.

We also analyze the impact of changing another aspect of patent policy, that is, patentability requirements. In order to be patentable an innovation must be sufficiently novel (not already in the public domain), non-obvious (to a person with ordinary skills in the particular field), and useful (to have at least one application). The relevant requirements vary across jurisdictions and are currently stricter in the EU than in the U.S. (Eckert and Langinier, 2014). In the spirit of Crampes and Langinier (2009), we model the patentability requirement as a minimum investment threshold level that must be satisfied. We then vary this investment threshold to examine whether a stricter patentability requirement fosters more green innovation. ${ }^{7}$

We also contribute to the green innovation literature by incorporating environmentally friendly consumers in our model. The increasing environmental consciousness of citizens globally is reflected in widely used eco-labeling schemes internationally. ${ }^{8} \mathrm{~A}$ few papers study optimal final disposition.

${ }^{7}$ Some studies have illustrated that strong Intellectual Property Rights may not necessarily enhance innovation (Green and Scotchmer, 1995; Gallini, 2002; Bessen and Maskin, 2009). Even though in a static world (single innovation), patents of appropriate scope can encourage innovations (Klemperer, 1990; Gilbert and Shapiro, 1990), this is no longer the case when the cumulative nature of innovation is accounted for. In the case of cumulative innovations, strict patentability requirement may even discourage follow-on innovations (Scotchmer, 1991). The prospect of being imitated inhibits inventors in a static world but, in a dynamic world, imitators can benefit both the original inventor and society (Bessen and Maskin, 2009). In our paper, we abstract away from these issues and present an alternative mechanism through which stronger patentability requirements affect innovation.

${ }^{8}$ For example, in countries like Sweden about $50 \%$ of the market share for certain products consists of the 
environmental policies in the presence of environmentally friendly consumers, but they do not address green innovation (Arora and Gangopadhyay, 1995; Cremer and Thisse, 1999; MoragaGonzalez and Padron-Fumero, 2002; Bansal and Gangopadhyay, 2003; Lombardini-Riipinen, 2005; Bansal, 2008). It is important to include environmentally friendly consumers since this might modify the role of emission taxes in inducing innovation. This is because, as the product becomes cleaner, the green conscious consumers demand more of it thereby mitigating the effect of emission taxes in reducing emissions. ${ }^{9,10}$ Our model is applicable to a wide variety of products, the production processes of which are becoming cleaner. Consider for example, products such as toys, furniture and packaging, the "greenness" of which may depend on the proportion of inputs used in the production process that are recycled. Recent evidence suggests that the volume of recycling has been increasing. ${ }^{11}$ Hence the importance of analyzing how behavioural responses of environmentally friendly consumers to such cleaner production processes that generate the "rebound-like effect" in our model, affect the functioning of policies such as emission taxes and environmentally friendly variant. Green marketing is also frequently used to influence consumer behavior in transportation and electricity markets (Kraftborsen, 2001).

${ }^{9}$ This is reminiscent of the "rebound effect" whereby as a product becomes more energy efficient, demand for the product increases (see Gillingham et al., 2016, for a survey). At the same time, we note that the channel through which the demand increases in our model is different from the rebound effect that is discussed in the energy efficiency literature. While in the latter, the increase in demand results from improved energy efficiency reducing the price of the good or the price of using the good, the "rebound-like effect" in our model is driven by environmentally friendly behaviour of consumers.

${ }^{10}$ Another difference between the rebound effect that is discussed in the energy efficiency literature, and the "rebound-like effect" in our model is that while the former is applicable to scenarios where innovation leads to a cleaner consumption process, our model is applicable to scenarios where innovation leads to a cleaner production process associated with a given product. For example, replacing a gasoline powered vehicle with a hybrid car reduces the pollution externality related to the consumption of the good (in this case, the pollution arising from driving). This paper, on the other hand, focuses on the case where the production process of the good causes less pollution. By focusing on the production related pollution externalities, rather than consumption related ones, we abstract away from cases where increasing demand caused by the availability of a cleaner product leads to less pollution, as when a gasoline powered vehicle is replaced by a consumer with a hybrid car.

${ }^{11}$ See Figures A1 to A2 in Appendix A which illustrate the increasing volume of recycling over time. For example, over the period 2005-2016, the volume of recycling in Europe increased by $34 \%$. 
patents for green innovation. ${ }^{12}$

We model the market for a product, the production of which causes pollution. We assume that the implementation of a cleaner technology results in a lower emission per unit of output ratio (similar to, for example, Benchekroun and Ray Chaudhuri, 2014, 2015). Moreover, similar to Bansal and Gangopadhyay (2003) and Ibañez and Grolleau (2008), we assume that the product is vertically differentiated in terms of its emission-output ratio with green conscious consumers preferring products with lower emission-output ratios. On the demand side, we allow consumers to be heterogeneous in terms of their degree of environmental friendliness. Our framework has two stages where, in the first stage, an incumbent monopolist decides its level of investment in $\mathrm{R} \& \mathrm{D}$, and in the second stage, it chooses the price of its product. The monopolist faces potential entry if it does not innovate. We assume that investment by the firm reduces the emission-output ratio. If the innovation is patented, the firm effectively behaves as a monopolist when setting its price in the second stage. If the innovation does not satisfy the patentability requirement, the firm cannot patent it and faces Bertrand competition in the second stage since entry occurs and rival firms are assumed to have free access to the new technology.

Within this setting, our main findings are two-fold.

The first set of results shows that, in the absence of green consumers, the introduction of patents may result in a paradoxical result whereby increasing the emission tax beyond a certain threshold makes the innovation unprofitable, and thereby leads to a discrete increase in the emission level. By introducing a fixed cost of obtaining patents, we obtain that increasing the emission tax beyond a certain threshold makes the innovation unprofitable. At this tax threshold, without the innovation, the emission level increases discretely to the competitive emission level, causing the paradox. Reducing the patenting cost, e.g., by fast-tracking green patents, decreases the likelihood that this paradox occurs. In the presence of green consumers, this paradox occurs only within an intermediate range of tax rates. This is because, at very

\footnotetext{
${ }^{12}$ The rebound effect as captured in the existing energy efficiency literature is an important phenomenon. Although the "rebound-like effect" that we capture in our model has not yet received as much attention in the existing literature as the traditional rebound effect, given its potential empirical relevance, it is useful to examine its policy implications.
} 
high tax rates, the emission level in the competitive equilibrium that occurs in the absence of innovation, is lower than that with innovation. If innovation occurred, demand from green consumers would increase thereby increasing the emission level above that in the competitive case without innovation. Thus, at very high tax rates, a lower emission level is reached despite a tax increase that makes the innovation unprofitable. It follows that, at sufficiently high tax rates, reducing patenting costs in order to induce innovation ends up increasing the emission level in the presence of green consumers. The lowest level of emissions, in the presence of green consumers, is therefore reached with very high emission tax rates combined with high patenting costs.

The second set of results shows that the traditional policy tools of increasing green investment and thereby reducing emissions through stricter emission taxes and patentability requirements may become less effective as society becomes more environmentally friendly, with consumers rewarding marginal reductions in emission-ouput ratios of production processes. More specifically, while for a sufficiently small fraction of green consumers we retrieve the expected result found in much of the literature surrounding the Porter hypothesis that a higher emission tax increases green investment, this result is reversed if the fraction of green consumers rises to a level such that investment in green technologies results in more emissions. These results are driven by the "rebound-like" effect introduced by the green consumers. We also show that a stricter patentability requirement is only effective at reducing emissions as long as the fraction of green consumers is sufficiently small. Finally, we show that as long as the fraction of green conscious consumers is sufficiently low, the firm underinvests relative to the socially optimal level for a sufficiently low emission tax and overinvests for a sufficiently high emission tax. However, the gap between the socially efficient and privately optimal levels of investment steadily reduces as the fraction of green conscious consumers increases, until this result is reversed when this fraction becomes sufficiently large. Thus, further research seems warranted regarding the policies to reduce emissions through green innovation and also regarding the type of information to distribute to consumers.

We extend our analysis to a duopoly setting, where a single firm decides to invest in a cleaner technology and patent its innovation in order to attract green consumers. Our results in the 
duopoly setting remain qualitatively similar to those derived in the monopoly setting.

The paper is organized as follows. In Section 2, we present our model. In Section 3, we present the benchmark case without green consumers. In Section 4, we derive the equilibrium and policy implications for the case with green consumers. Section 5 presents an extension of the model with a duopoly case. Section 6 presents our concluding remarks. All proofs have been relegated to Appendices A and B.

\section{The Model}

We consider a two-stage model in which a firm sells a final good to consumers in a competitive market, the production of which is polluting and has a marginal cost, $c$. The emission of the pollutant generated per unit of production is given by:

$$
\gamma \equiv \frac{e}{q}
$$

where $e$ denotes emission and $q$ denotes output. The firm can invest $I_{G}$ to reduce the emissionoutput ratio, $\gamma$. Thus, $\gamma$ is a function of $I_{G}$, where $\gamma\left(I_{G}\right)$ is such that $\gamma^{\prime}\left(I_{G}\right)<0, \gamma^{\prime \prime}\left(I_{G}\right)>0$, $\gamma(0)=\gamma_{H}$ and $\lim _{I_{G} \rightarrow \infty} \gamma\left(I_{G}\right)=\gamma_{L}>0$. The higher is $I_{G}$, the greener the product. For notational convenience, henceforth we do not mention the argument of the function $\gamma$.

\subsection{The demand side}

The demand side consists of a continuum of $N$ consumers. Each of them buys either 0 or 1 unit of the good. There exists a fraction $\lambda$ of 'green conscious' consumers, whose utility is increasing in the "greenness" of the product, that is, decreasing in $\gamma$, and a fraction $(1-\lambda)$ of "non-green conscious' consumers, whose utility is independent of the greenness of the product.

Let $G$ denote the degree of environmental friendliness of a consumer, with $G$ being uniformly distributed over the interval $[\underline{G}, \bar{G}]$ with $\underline{G}>0$. We assume that consumers can observe how green a product is. Within this context, this is equivalent to assuming that consumers can observe $\gamma{ }^{13}$ We normalize $N$ such that $N=1$, and assume that $\bar{G}-\underline{G}=1$. Let $P(e)$ denote

\footnotetext{
${ }^{13}$ This is a relevant scenario to consider in the presence of effective eco-labeling programs.
} 
the pollution damage to each consumer, which is a function of total emissions, e. Following Ibañez and Grolleau (2008), we assume that the pollution level generated by total production is exogenous to each consumer, regardless of his consumption level. Let $p$ denote the product price.

A green conscious consumer has the following utility function:

$$
U_{G}=\left\{\begin{array}{cc}
v-G \gamma-p-P(e) & \text { from buying the product } \\
-P(e) & \text { from not buying }
\end{array}\right.
$$

The term $-G \gamma$ in (2) reflects that the greener the product, that is, the lower is $\gamma$, the better off the green conscious consumer. Also, $v$ represents the gross utility of consuming one unit of the good. A green conscious consumer does not buy the product if $v-G \gamma-p<0$.

A non-green conscious consumer has the following utility function:

$$
U_{N G}=\left\{\begin{array}{cc}
v-p-P(e) & \text { from buying the product } \\
-P(e) & \text { from not buying }
\end{array}\right.
$$

Henceforth, we assume that $P(e)=e$.

From (3), it follows that a non-green conscious consumer buys the good as long as $v \geq p$. Therefore, if $v<p$, neither non-green conscious consumers nor green conscious consumers buy the good. If $p=v$, only non-green conscious consumers buy the good. When $p<v$, nongreen conscious consumers always buy the good whereas a green conscious consumer with a degree of environmental friendliness $G$ buys the good as long as $v-G \gamma-p \geq 0$. There exists a green conscious consumer $\widetilde{G}$ who is indifferent between buying the good or not such that $v-\gamma \widetilde{G}-p-P(e)=-P(e)$ or $\widetilde{G}=(v-p) / \gamma$. As long as $\underline{G}<\widetilde{G}<\bar{G}$, some, but not all, green conscious consumers buy the good. Thus, it follows that the demand function, $D(p)$, is given by:

$$
D(p)= \begin{cases}1 & \text { if } p \leq v-\gamma \bar{G} \\ \lambda\left(\frac{v-p}{\gamma}-\underline{G}\right)+(1-\lambda) & \text { if } v-\gamma \bar{G}<p<v-\gamma \underline{G} \\ (1-\lambda) & \text { if } v-\gamma \underline{G} \leq p \leq v \\ 0 & \text { if } p>v\end{cases}
$$




\subsection{Policy Tools}

We consider a combination of policy tools. On the $R \& D$ side, we model a patenting policy for green innovations. On the environmental side, we assume that the firm must pay a tax, $\tau$, per unit of emission. Thus, the tax bill faced by the firm is given by $\tau \gamma D(p)$, where, by $(1), \gamma D(p)$ represents the emissions generated by the firm.

The patent policy is such that the firm must discover a sufficiently novel innovation to be able to obtain a patent. We assume that novelty of the innovation is increasing in the investment level. Recall that $\gamma^{\prime}\left(I_{G}\right)<0$, such that a higher investment level reduces the emission-output ratio. Thus, there exists a threshold $\gamma_{P}$, corresponding to investment level $I_{P G}$, where $\gamma_{L}<\gamma_{P}<\gamma_{H}$, such that a patent is only granted if an innovation reduces $\gamma$ below $\gamma_{P} \cdot{ }^{14}$ Therefore, the firm must invest $I_{G} \geq I_{P G}$ in order to ensure that $\gamma \leq \gamma_{P}$. We consider weak and strong patentability requirements, representing different levels of $I_{P G}$, as defined in Section 4.3 by Definition 1.

In order to obtain a patent, the firm must also incur an exogenously given $\operatorname{cost} C_{P G}$, which is broadly defined to include a monetary fee payable by the firm to the patent office, the opportunity cost in terms of lost profits incurred while waiting for the patent to be granted, as well potential litigation costs for enforcing the patent. There are several ways in which policy makers may reduce $C_{P G}$, including by implementing a fast-track patent system for green technologies that reduces the patent application processing time for green innovations.

Once a patent is granted and the firm starts producing at a lower emission output ratio, this lower value of $\gamma \leq \gamma_{P}$ becomes the new technology standard for the industry that is enforced by regulators. That is, we implicitly assume that no rival firm can enter the industry if its production results in a higher $\gamma$. If the innovation does not satisfy the relevant patentability requirements, the firm cannot patent it, and faces Bertrand competition in the second stage. We note that such technology-based standards are widely used for environmental regulation. ${ }^{15}$

\footnotetext{
${ }^{14}$ We implicitly assume that the novelty of the innovation is assessed by an experienced patent examiner who is able to evaluate whether the innovation meets the patentability requirement.

${ }^{15}$ For example, in Germany technology standards were used to reduce sulphur-dioxide emissions. In Canada, under the Canadian Enviromental Protection Act, technology standards apply to a number of industries including the energy sector, pulp and paper mills, and mineral smelters.
} 
Moreover, technology standards are widely used as a trade barrier to keep foreign competition out of domestic markets. ${ }^{16}$ In order to implement technology standards, the regulator prescribes certain technologies, design standards, engineering standards or input standards which require potential polluters to use inputs and production processes meeting specific conditions. Typically, technology standards specify that polluters use the "best available technology" (BAT), ${ }^{17}$ implying that, subsequent to an innovation which improves the best available technology in a given industry regulated by technology standards, firms with dirtier technologies would be unable to enter the industry.

\subsection{Timing}

The timing of the game is as follows. There are two stages. In the first stage of the game, the firm decides the level of investment in the green technology, $I_{G} \cdot{ }^{18}$ Once an innovation has been discovered, the firm decides whether to patent it. ${ }^{19}$ In the second stage, the firm chooses the price of the product it offers, $p$.

We solve for the equilibrium investment level and price through backward induction. For a given level of investment, we first determine the pricing strategy of the firm. Then, we determine the level of investment at the equilibrium.

As a benchmark case, we first consider the scenario where there exist only non-green conscious consumers (i.e., $\lambda=0$ ) in Section 3. Next, we enrich our analysis by considering that both green and non-green conscious consumers exist (i.e., $0<\lambda \leq 1$ ), in Section 4 .

\footnotetext{
${ }^{16}$ For further details, please refer to: https://www.international.gc.ca/trade-agreements-accordscommerciaux/topics-domaines/goods-produits/barriers.aspx?lang=eng

${ }^{17}$ See, for example, Field and Olewiler (2011).

${ }^{18} \mathrm{An}$ alternate interpretation of the investment level, $I_{G}$, in our model is that it represents the cost incurred by the firm for obtaining the innovation.

${ }^{19}$ We make the simplifying assumption that an innovation will be discovered with probability 1 . If we alternatively assumed that an innovation will be discovered with an exogenously given probability less than 1, this would make the notation and analysis more cumbersome without generating any new insights.
} 


\section{Benchmark Case: Non-Green Conscious Consumers}

In this section, we present the scenario without any green consumers, that is, $\lambda=0$. In the second stage, we have monopoly pricing, whereby the profit maximizing price is given by $p=v$. From (4), it follows that, for $\lambda=0$, the demand is given by $D=1$. Thus, the emission level is given by $e=\gamma\left(I_{G}\right)$, and the profit is given by $(v-c-\tau \gamma)$. Recall that $c$ denotes the marginal cost of production.

In the first stage, the firm's optimization problem is given by:

$$
\left\{\begin{array}{c}
\underset{I_{G}}{\operatorname{Max}}\left[v-c-\tau \gamma\left(I_{G}\right)-I_{G}\right] \\
\text { s.t. } \gamma \leq \gamma_{P} \equiv \gamma\left(I_{P G}\right)
\end{array}\right.
$$

Let $I^{N G}$ be the solution of the unconstrained program (i.e., $-\tau \gamma^{\prime}\left(I^{N G}\right)=1$ ); the firm therefore chooses the optimal investment $I_{G}^{N G}=\max \left\{I^{N G}, I_{P G}\right\}$. As long as the unconstrained investment is large enough $\left(I^{N G}>I_{P G}\right)$ so that the firm optimally chooses to reduce its emission-output ratio sufficiently, it is not constrained by the patentability requirement. However, if $I^{N G}<I_{P G}$, in order to patent its innovation, the firm has to increase its investment to $I_{P G}$, which is beyond its unconstrained optimal investment.

The unconstrained optimization investment level $I^{N G}$ is increasing with the $\operatorname{tax} \tau$ so that the emission level is decreasing. However, for very low values of $\tau$ (in the extreme case if $\tau=0$ ), the firm has very little (no) incentive to invest, so that $0 \leq I^{N G}<I_{P G}$ is always satisfied.

Consider two threshold levels of emission tax, $\hat{\tau}_{1}$ and $\hat{\tau}_{2}$, such that $\gamma_{P}=e\left(I^{N G}\left(\hat{\tau}_{1}\right)\right)$ and $C_{P G}=v-c-\hat{\tau}_{2} \gamma\left(I^{N G}\left(\hat{\tau}_{2}\right)\right)-I^{N G}\left(\hat{\tau}_{2}\right)$. Our first set of findings concerning the emission level is summarized in Proposition 1.

Proposition 1 The emission level, with $\lambda=0$

(i) is given by $\gamma_{P}$ for $\tau \in\left[0, \hat{\tau}_{1}\right)$,

(ii) decreases due to marginal increases in the tax rate at any $\tau \in\left[\hat{\tau}_{1}, \hat{\tau}_{2}\right)$,

(iii) increases discretely due to an increase in the tax rate from any $\tau<\hat{\tau}_{2}$ to any $\tau>\hat{\tau}_{2}$.

The findings listed in Proposition 1 are illustrated in Figure 1 below (see Appendix A for the explanation regarding the shapes of the functions in all the figures). 


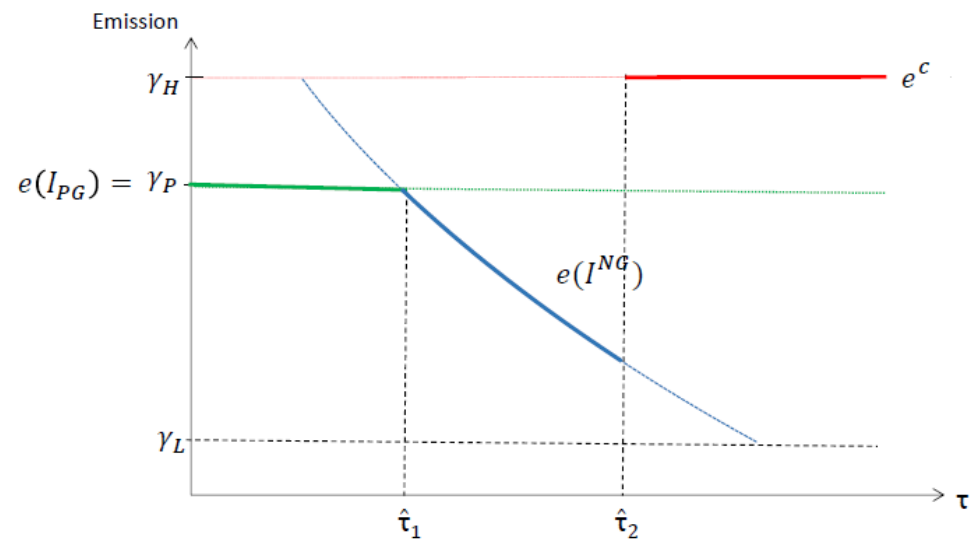

Figure 1: Profit maximizing emission levels when $\lambda=0$

For a sufficiently low tax rate, that is, $\tau \in\left[0, \hat{\tau}_{1}\right)$, the firm invests the minimum amount required to obtain a patent, that is, $I_{P G}$. For $\tau \in\left[\hat{\tau}_{1}, \hat{\tau}_{2}\right)$, the firm has an incentive to invest more than this minimum level since $\gamma\left(I^{N G}\right)<\gamma_{P}$. We note that the firm only invests as long as

$$
v-c-\tau \gamma\left(I^{N G}\right)-I^{N G}-C_{P G}>0
$$

which is not satisfied for $\tau>\hat{\tau}_{2}$. Thus, for $\tau>\hat{\tau}_{2}$, the firm does not invest and Bertrand competition results in emission level given by $e^{c}=\gamma_{H}$.

Proposition 1 highlights the first contribution of our paper, which is to show that the introduction of patents may result in a paradoxical result whereby increasing the emission tax beyond a certain threshold leads to a discrete increase in the emission level. Moreover, it follows from (5) that the higher the patenting cost, $C_{P G}$, the greater the range of taxes for which the paradox occurs.

Corollary 1 The lower the patenting cost, $C_{P G}$, the less likely that the emission level increases as emission tax increases.

A direct policy implication of Corollary 1 is that if $C_{P G}$ is reduced by any means, such as fast-tracking green patents, the less likely that this paradox occurs and the more effective are emission taxes at inducing green innovation. This follows from the fact that the threshold $\hat{\tau}_{2}$ is 
decreasing in $C_{P G}$, by definition. ${ }^{20}$

In the following section, we examine whether the results summarized in Proposition 1 and Corollary 1 carry over to the scenario with green consumers.

\section{The Equilibrium with Green Consumers}

In this section, we continue our analysis with the general model introduced in Section 2 with green consumers, that is, $0<\lambda \leq 1$.

\subsection{Second Stage: Monopoly Pricing}

We begin with the second stage, and analyze the pricing strategy of the firm. Assuming that the innovation has been patented, in the second period the firm solves the following:

$$
\max _{p} \Pi \equiv(p-c) D(p)-\tau \gamma D(p),
$$

where the demand is given by (4). Due to the discontinuities in the demand function, depending on the chosen price, the firm will face either all green and non-green consumers, some green consumers and all non-green consumers, or only non-green consumers. The only relevant cases for our analysis are those where non-green consumers and some green consumers consume, and only non-green consumers buy the good. Therefore, we will only consider a constellation of parameters such that the cases where demand is inelastic at 1 or at 0 are ruled out.

If only non-green consumers buy the good, the demand is $(1-\lambda)$ such that the firm sets its price at $v$ and, therefore, the profit is given by:

$$
\Pi^{N G}=(v-c-\tau \gamma)(1-\lambda)
$$

where the emission level is thus $e=\gamma(1-\lambda)$.

If some green conscious consumers and all non-green conscious consumers buy the good, the profit-maximizing price is given by:

$$
p^{m}\left(I_{G}\right)=\frac{1}{2}(v+c-\gamma(\underline{G}-\tau))+\frac{1-\lambda}{2 \lambda} \gamma,
$$

\footnotetext{
${ }^{20}$ Recall that $C_{P G}=v-c-\hat{\tau}_{2} \gamma\left(I^{N G}\left(\hat{\tau}_{2}\right)\right)-I_{P G}$. Since $I_{P G}$ is constant and $v-c-\tau \gamma\left(I^{N G}\right)$ is decreasing in $\tau$, we have that an increase in $C_{P G}$ must correspond to a decrease in $\hat{\tau}_{2}$.
} 
and the second order condition is satisfied since $\partial^{2} \Pi / \partial p^{2}=-2 \lambda / \gamma<0$.

In order to get demand from both types of consumers, we need to ensure that $p^{m}\left(I_{G}\right)<v-\gamma \underline{G}$ or, equivalently, that $\lambda>\underline{\lambda}$, where

$$
\underline{\lambda} \equiv \frac{\gamma}{\gamma+v-c-\gamma(\underline{G}+\tau)}>0 \text {. }
$$

We show that $\underline{\lambda}<1$ if $\gamma<(v-c) /(\underline{G}+\tau)$. In order for this condition to be satisfied for any $\gamma$, we assume that

$$
\gamma_{H}<\frac{v-c}{\underline{G}+\tau}
$$

so that for any $\gamma<\gamma_{H}$, we have $\underline{\lambda}<1$.

From (8), it follows that the demand is given by:

$$
D=\frac{\lambda}{2 \gamma}[v-c-\gamma(\underline{G}+\tau)]+\frac{1}{2}(1-\lambda) \text {. }
$$

For values of $\lambda>\underline{\lambda}$, the demand function, evaluated at the price $p^{m}\left(I_{G}\right)$, is decreasing in the marginal cost, $c$, and is increasing in the valuation, $v$. Since $\partial D / \partial \gamma=-\lambda(v-c) / 2 \gamma^{2}<0$, we have that the demand is increasing in $I_{G}$. As the investment in the green technology increases, the demand increases as the product becomes greener.

To ensure that some but not all green conscious consumers buy the product (and rule out the case of inelastic demand 1), we further assume that

$$
\gamma_{L}>\frac{v-c}{\bar{G}+\tau}
$$

Therefore, assumptions (A1) and (A2) ensure that the extreme cases of $D=0$ and $D=1$ are avoided, since these cases would lead to discontinuities. ${ }^{21}$

By substituting (8) and (9) into (6), when both non-green and green conscious consumers buy, we obtain the net profit of the firm in the second stage as the following:

$$
\Pi^{m}=\frac{\lambda}{4 \gamma}\left[v-c-\gamma(\underline{G}+\tau)+\frac{1-\lambda}{\lambda} \gamma\right]^{2} .
$$

\footnotetext{
${ }^{21}$ More specifically, the condition $\gamma_{H}<(v-c) /(\underline{G}+\tau)$ implies that the least environmentally friendly consumer has a positive demand for the dirtiest good ensuring that $D \neq 0$ for $\lambda=1$, and together with (A2) ensures that $D \neq 0$ for all $\lambda>\underline{\lambda}$. Moreover, the condition $\gamma_{L}>(v-c) /(\bar{G}+\tau)$ implies that the most environmentally friendly consumer does not buy the cleanest good ensuring that $D \neq 1$ for $\lambda=1$. Also, $\gamma_{L}>(v-c) /(\bar{G}+\tau)$ is a sufficient condition to ensure that $\widetilde{G}<\bar{G}$ for all $\lambda>\underline{\lambda}$. Thus, Assumptions (A1) and (A2) together ensure that $\underline{G}<\widetilde{G}<\bar{G}$.
} 
Even if both types of consumers are willing to buy the good (i.e., for $\lambda>\underline{\lambda}$ ), the firm can either choose to serve only non-green consumers and set its price at $v$, or to serve all types of consumers by setting the price (8). The firm will choose to serve only non-green consumers if $\Pi^{N G}>\Pi^{m}$ where $\Pi^{N G}$ is defined by (7) and $\Pi^{m}$ by (10). This is equivalent to having $\lambda<\lambda_{1}$ where

$$
\lambda_{1} \equiv \frac{\gamma}{\gamma+v-c+\gamma(\underline{G}-\tau)-2(v-c-\gamma \tau)^{\frac{1}{2}}(\gamma \underline{G})^{\frac{1}{2}}} .
$$

See Appendix A for details of the derivation of $\lambda_{1}$. It is straightforward to show that $\lambda_{1}>\underline{\lambda}$ as long as (A1) is satisfied. Therefore, if the fraction of green consumers is small $(\lambda<\underline{\lambda})$, only non-green consumers demand the product. However, even if green consumers are willing to buy the good, the firm might set its price at $v$ such that only non-green consumers will purchase. Thus, for $\lambda \in\left[\underline{\lambda}, \lambda_{1}\right]$, only non-green consumers buy as the price is too high for green consumers to buy. For $\lambda \in\left[\lambda_{1}, 1\right]$, both types of consumers buy the good at the price given by (8).

To summarize, when $\lambda \in\left[\lambda_{1}, 1\right]$, the firm chooses the monopoly price (8) in order to serve all types of consumers. When $\lambda \in\left[0, \lambda_{1}\right]$, only non-green consumers buy the good at price $v$.

Thus, for $\lambda \in\left[\lambda_{1}, 1\right]$, from (9) it follows that for any $I_{G}$, the emission level of the firm is given by:

$$
e^{m}\left(I_{G}\right)=\frac{\lambda}{2}[v-c-\gamma(\underline{G}+\tau)]+\frac{\gamma}{2}(1-\lambda) .
$$

Let

$$
\lambda_{G} \equiv \frac{1}{\underline{G}+\tau+1} .
$$

The following Lemma follows directly from (12) (the proof is provided in Appendix A).

Lemma 1 The emission level, $e^{m}\left(I_{G}\right)$,

(i) is decreasing in the emission tax, $\tau$, and

(ii) is decreasing in $I_{G}$ for $\lambda \in\left(\lambda_{1}, \lambda_{G}\right]$, and increasing in $I_{G}$ for $\lambda \in\left(\lambda_{G}, 1\right]$.

In the absence of many green-conscious consumers, i.e., $\lambda \in\left(\lambda_{1}, \lambda_{G}\right]$, we obtain the standard result that an increase in investment in green technology results in less emissions. However, the rate of decrease of emissions steadily reduces as $\lambda$ increases (since $\frac{\partial^{2} e^{m}\left(I_{G}\right)}{\partial I_{G} \partial \lambda}=-\frac{1}{2}(\underline{G}+\tau+1) \frac{d \gamma}{d I_{G}}>$ 
0) until it becomes positive for $\lambda>\lambda_{G}$. Thus, Lemma 1(ii) implies that private investment by firms in green technologies may lead to more emissions if the fraction of green conscious consumers is sufficiently large, i.e., $\lambda>\lambda_{G}$. This is because, from (9), we have that demand is increasing in $I_{G}$ since as the product becomes cleaner, the environmentally friendly consumers demand more of it. ${ }^{22}$ Moreover, Lemma $1(i)$ states that regardless of the impact of $I_{G}$ on the emission level, an increase in the emission tax rate decreases the emission level. Lemma $1(i)$ and $(i i)$ together imply that when firms invest in green technologies in the presence of green consumers who increase their demand for cleaner products, it becomes necessary to implement environmental regulation, such as an emission tax to ensure a reduction in the emission level.

Figure 2 illustrates, in more detail, the impact of increasing investment in green technology on emissions.

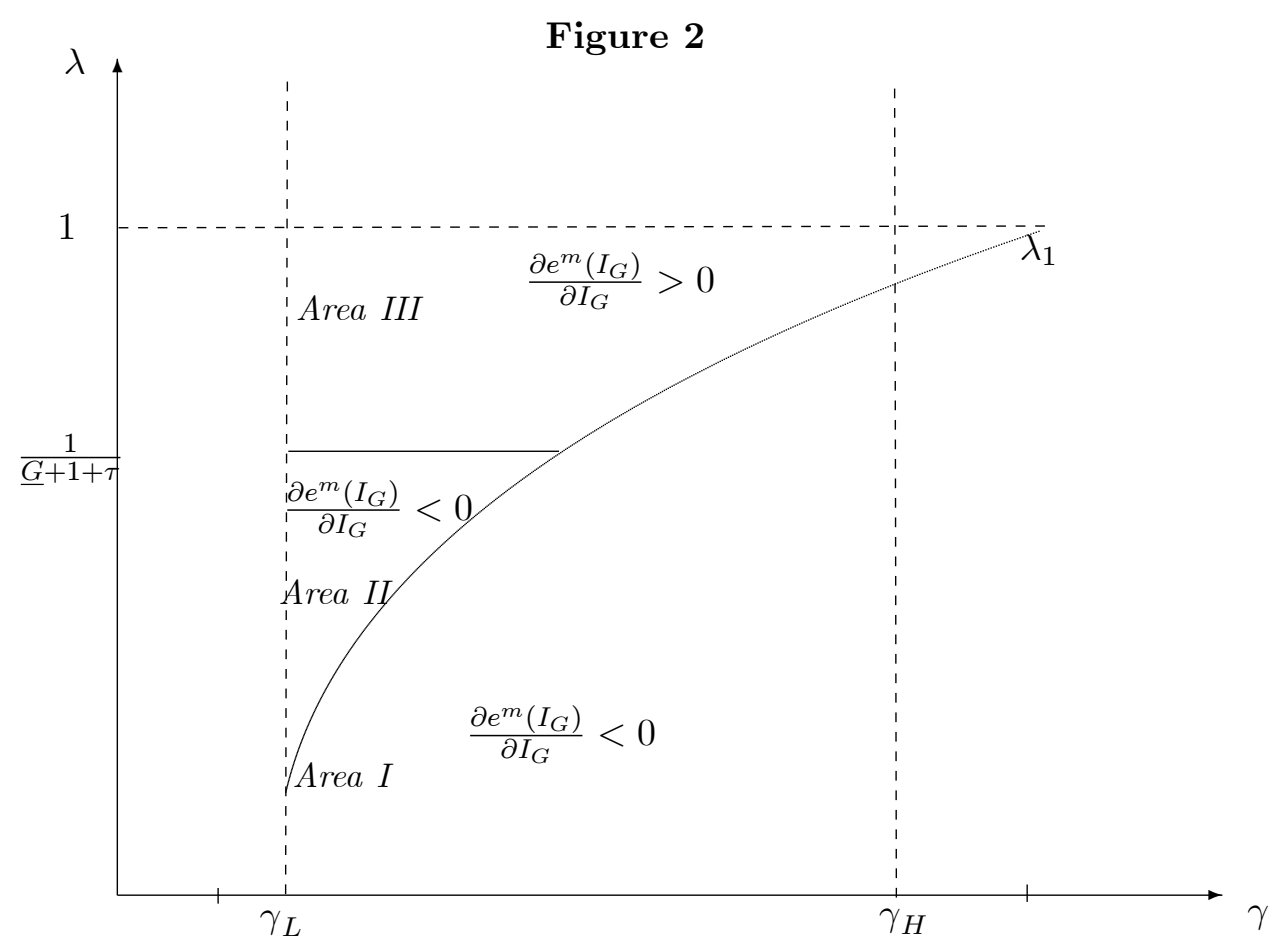

In Figure 2, in Areas I and II, we have $\frac{\partial e^{m}\left(I_{G}\right)}{\partial I_{G}}<0$, and in Area III, we have $\frac{\partial e^{m}\left(I_{G}\right)}{\partial I_{G}}>0$. Area I represents the combinations of $\gamma$ and $\lambda$ for which the monopolist chooses to serve only

\footnotetext{
${ }^{22}$ This is reminiscent of the rebound effect, as explained earlier in Footnote 9.
} 
non-green conscious consumers by setting $p=v$. This occurs for $\lambda \in\left(0, \lambda_{1}\right)$. In fact, for $\lambda \in(0, \underline{\lambda})$ only non-green conscious consumers demand the good, while for $\lambda \in\left(\underline{\lambda}, \lambda_{1}\right)$, some green conscious consumers are willing to buy, but the firm chooses to set a price that is too high for them to buy. In Area I, the emission level is given by:

$$
e^{m}\left(I_{G}\right)=\gamma(1-\lambda)
$$

and thus,

$$
\frac{\partial e^{m}\left(I_{G}\right)}{\partial I_{G}}=\gamma^{\prime}(1-\lambda)<0 .
$$

For $\lambda>\lambda_{1}$, the monopolist chooses $p^{m}$ and serves both green and non-green consumers (Areas II and III in Figure 2). The emission level in Areas II and III is given by (12). Area II

represents the case where $\lambda \in\left(\lambda_{1}, \lambda_{G}\right)$ such that $\frac{\partial e^{m}\left(I_{G}\right)}{\partial I_{G}}<0$, as per Lemma 1, and Area III represents the case where $\lambda>\lambda_{G}$ such that $\frac{\partial e^{m}\left(I_{G}\right)}{\partial I_{G}}>0$, as per Lemma 1 .

\subsection{First Stage: Profit-Maximizing Investment}

In the first stage of the game, the firm chooses the investment level in green technology $I_{G}$ that solves the following problem:

$$
\left\{\begin{array}{cc} 
& \underset{I_{G}}{\operatorname{Max} \Pi^{m}\left(I_{G}\right)-I_{G}} \\
\text { s.t. } & \gamma\left(I_{G}\right) \leq \gamma_{P} \equiv \gamma\left(I_{P G}\right)
\end{array}\right.
$$

where the profit $\Pi^{m}\left(I_{G}\right)$ is defined by (10) for $\lambda \in\left(\lambda_{1}, 1\right] .{ }^{23}$ Let $I_{G}^{m}$ be the solution of the unconstrained optimization problem (i.e., $\left.I_{G}^{m}=\arg \max \Pi^{m}\left(I_{G}\right)-I_{G}\right)$ such that it is the solution of the following first order condition:

$$
-\gamma^{\prime}\left(I_{G}\right)[\underbrace{\frac{\lambda}{4}\left(\left(\frac{v-c}{\gamma\left(I_{G}\right)}\right)^{2}-(\underline{G}+\tau)^{2}\right)}_{(i)}+\underbrace{\frac{(1-\lambda)}{2}(\underline{G}+\tau)-\frac{1}{4} \frac{(1-\lambda)^{2}}{\lambda}}_{(i i)}]=1 .
$$

We note that the left-hand side of equation (13) is positive such that (13) is satisfied as long as Assumptions (A1) and (A2) hold (see Appendix A for details). The first term (i) of (13) captures the change in demand of green consumers as investment changes marginally, whereas the second term $(i i)$ captures the change in demand of non-green consumers. The second term

\footnotetext{
${ }^{23}$ The case where $\lambda \in\left[0, \lambda_{1}\right)$ is similar to the benchmark case with only non-green consumers.
} 
can push the investment level down since it can be negative. To obtain an interior maximum solution, we further assume that

$$
\gamma^{\prime \prime}\left(I_{G}\right)>-\lambda \frac{(v-c)^{2}}{2}\left(\frac{\gamma^{\prime}\left(I_{G}\right)}{\gamma\left(I_{G}\right)}\right)^{3}
$$

such that the second order condition is satisfied (see Appendix A for details).

The solution of the constrained problem is thus $I^{m} \equiv \operatorname{Max}\left\{I_{G}^{m}, I_{P G}\right\}$. Indeed, as long as the constraint is always satisfied, we have $I_{G}^{m}>I_{P G}$ and thus the firm chooses its optimal investment level. When the constraint binds, the firm must invest $I_{P G}>I_{G}^{m}$.

\subsection{Policy Implications}

Having solved for the equilibrium of the model, we now turn to analyzing the effect of changes in emission tax and patent policies. In order to do so, we first define "weak" and "strong" patentability requirements.

Definition $1 A$ "weak" patentability requirement is defined to be $I_{P G} \leq I_{G}^{m}$ for $\tau=0$, and a "strong" patentability requirement is defined to be $I_{P G}>I_{G}^{m}$ for all $\tau \geq 0$.

By Definition 1, a strong patentability requirement means that no matter what the emission tax level, the unconstrained optimal investment level of the monopolist, $I_{G}^{m}$, is always below the required investment level to obtain a patent, $I_{P G}$. A weak patentability requirement means that in the absence of any emission tax, the monopolist naturally invests more than the minimum required to obtain a patent. However, $I_{G}^{m}$ is decreasing with the tax level, ${ }^{24}$ so that as the tax increases we may have $I_{P G} \geq I_{G}^{m}$.

In what follows, we consider that the fraction of green conscious consumers is such that $\lambda>\lambda_{1}$. When the patentability requirement is weak, we begin by focusing on the case where the firm chooses to invest $I_{G}^{m}$ in the first stage, patents its green innovation and sets the price $p\left(I_{G}^{m}\right)$ in the second stage as long as $\Pi^{m}\left(I_{G}^{m}\right)-I_{G}^{m}-C_{P G}>0 .{ }^{25}$

\footnotetext{
${ }^{24}$ See the section on comparative statics in Appendix 1 for further details. A summary of the results is provided in Table 1 in Appendix A.

${ }^{25}$ See Appendix 1 for comparative statics with respect to $c$ and $v$.
} 
Lemma 2 For a weak patentability requirement, investment in green technology, $I_{G}^{m}$,

(i) is increasing in the emission tax, $\tau$, and in $\underline{G}$, for $\lambda \in\left(\lambda_{1}, \lambda_{G}\right]$,

(ii) is decreasing in the emission tax, $\tau$, and in $\underline{G}$, for $\lambda \in\left(\lambda_{G}, 1\right]$.

Lemma 2 follows directly from (13) (see Appendix A for details of the calculations). In the absence of many green conscious consumers, i.e., $\lambda \in\left(\lambda_{1}, \lambda_{G}\right]$, we obtain the standard result that an increase in emission tax induces greater investment in green technology. However, the rate of increase of investment steadily reduces as $\lambda$ increases, until it becomes negative for $\lambda>\lambda_{G}$. This is because by Lemma 1, an increase in $I_{G}$ would be accompanied by an increase in emissions for $\lambda>\lambda_{G}$, and thereby, an increase in the tax bill facing the firm. Thus, for $\lambda>\lambda_{G}$, the firm chooses to decrease green investment when faced with a higher emission tax. Changes in $\underline{G}$ play a similar role to changes in $\tau$ in our model.

The intuition behind Lemma 2 is that while trying to lower the tax bill by increasing innovation, the firm faces a trade-off between lower emission per unit of good and higher demand. The former effect outweighs the latter when the fraction of green consumers is not too high, but the opposite is true when the fraction of green consumers becomes too large.

For $\lambda \in\left(\lambda_{G}, 1\right]$, an implication of Lemma 2 is that $I_{G}^{m}$ keeps falling as $\tau$ increases until we have $I_{P G}>I_{G}^{m}$. Let $\tilde{\tau}$ denote that level of the tax rate where $I_{G}^{m}=I_{P G}$. For $\tau>\tilde{\tau}$, the firm must invest more than $I_{G}^{m}$ in order to satisfy the patentability requirement. That is, the investment level is given by $I^{m} \equiv \operatorname{Max}\left\{I_{G}^{m}, I_{P G}\right\}$. Thus, the equilibrium price is given by $p=p^{m}\left(I^{m}\right)$. We summarize this finding in the following Lemma.

Lemma 3 If $C_{P G}<\Pi^{m}\left(I_{G}\right)-I_{G}$, the firm

(i) invests $I^{m} \equiv \max \left\{I_{P G}, I_{G}^{m}\right\}$ in the first period, patents its innovation and

(ii) chooses the price $p=p^{m}\left(I^{m}\right)$ as defined by (8) in the second period.

The quantity sold and the emission level in equilibrium are given by:

$$
q^{m}\left(I^{m}\right)=\frac{\lambda}{2 \gamma\left(I^{m}\right)}\left[v-c-\gamma\left(I^{m}\right)(\underline{G}+\tau)\right]+\frac{1}{2}(1-\lambda),
$$


and

$$
e^{m}\left(I^{m}\right)=\frac{\lambda}{2}\left[v-c-\gamma\left(I^{m}\right)(\underline{G}+\tau)\right]+\frac{1}{2}(1-\lambda) \gamma\left(I^{m}\right) .
$$

If $I_{P G}$ is larger, that is, the patentability requirement is strong as per Definition 1 , the firm must invest $I_{P G}$ for any $\tau \geq 0$ in order to satisfy the patentability requirement and will also set a higher price $p^{m}\left(I_{P G}\right)>p^{m}\left(I_{G}^{m}\right)$. It will invest as long as $\Pi^{m}\left(I_{P G}\right)-I_{P G}-C_{P G}>0$.

Thus far, we have presented the case where the firm invests in the green technology and patents it. However, the decision regarding whether to invest depends on the patenting cost, $C_{P G}$.

If the patenting cost is relatively small $\left(C_{P G}<\Pi^{m}\left(I_{P G}\right)-I_{P G}\right)$, the firm always invests, no matter how stringent the patent policy. If the patenting cost is very large, $\left(C_{P G}>\Pi^{m}\left(I_{G}^{m}\right)-I_{G}^{m}\right)$, the firm never invests. For intermediate values of the patenting cost $\left(\Pi^{m}\left(I_{P G}\right)-I_{P G}<C_{P G}<\right.$ $\left.\Pi^{m}\left(I_{G}^{m}\right)-I_{G}^{m}\right)$, a too stringent patentability requirement discourages the firm from investing, whereas a less strict patentability requirement induces the firm to invest. We summarize these findings in the following Lemma.

Lemma 4 The firm invests in the green technology

(i) if the patenting cost is small $\left(C_{P G}<\Pi^{m}\left(I_{P G}\right)-I_{P G}\right)$, or

(ii) if the patenting cost is higher $\left(\Pi^{m}\left(I_{P G}\right)-I_{P G}<C_{P G}<\Pi^{m}\left(I_{G}^{m}\right)-I_{G}^{m}\right)$, but the patentability requirement is not strong $\left(I_{P G} \leq I_{G}^{m}\right)$.

If the conditions in terms of $C_{P G}$, as per Lemma 4 , are not satisfied, the firm does not invest, such that Bertrand competition occurs in the second stage of the game with $\gamma=\gamma_{H}$. In this case, the price is given by $p^{c}=c+\gamma_{H} \tau$, the demand is given by $D\left(p^{c}\right)=\lambda\left(v-c-\gamma_{H}(\underline{G}+\tau)\right) / \gamma_{H}+(1-\lambda)$ and the emission level is given by:

$$
e^{c}=\lambda\left(v-c-\gamma_{H}(\underline{G}+\tau)\right)+\gamma_{H}(1-\lambda) .
$$

Lemmas 2, 3 and 4 are summarized in Figures 3 and 4 below. Using these Figures, we examine whether the paradox that occurs in the benchmark case without green consumers carries over 
to the case with green consumers: first under a weak patentability requirement (Figure 3), and then under a strong patentability requirement (Figure 4).

Figure 3 illustrates the equilibrium emission level as a function of $\tau$ under a weak patentability requirement if $\lambda>\left.\lambda_{G}\right|_{\tau=0}(=1 /(\underline{G}+1))$ and $\gamma_{H}<(v-c) / 2 \underline{G}$ (see Appendix A for details).

Let $\tau_{1}$ denote the threshold level of $\tau$ such that $e^{m}\left(I_{G}^{m}\right)=e^{m}\left(I_{P G}\right)$. Let $\tau_{2}$ denote the threshold level of $\tau$ such that $C_{P G}=\left.\Pi^{m}\left(I_{P G}\right)\right|_{\tau=\tau_{2}}-I_{P G}$. Let $\tau_{3}$ denote the threshold level of $\tau$ such that $e^{m}\left(I_{P G}\right)=e^{c}$. Let $\widehat{\tau}$ denote the threshold level of $\tau$ such that $e^{c}=0$. The top (red) function represents $e^{c}$, the curved (blue) one represents $e^{m}\left(I^{m}\right)$ when $I^{m}=I_{G}^{m}$ and the lower (green) one represents $e^{m}\left(I^{m}\right)$ when $I^{m}=I_{P G}$. The profit maximizing emission level is represented in bold. ${ }^{26}$

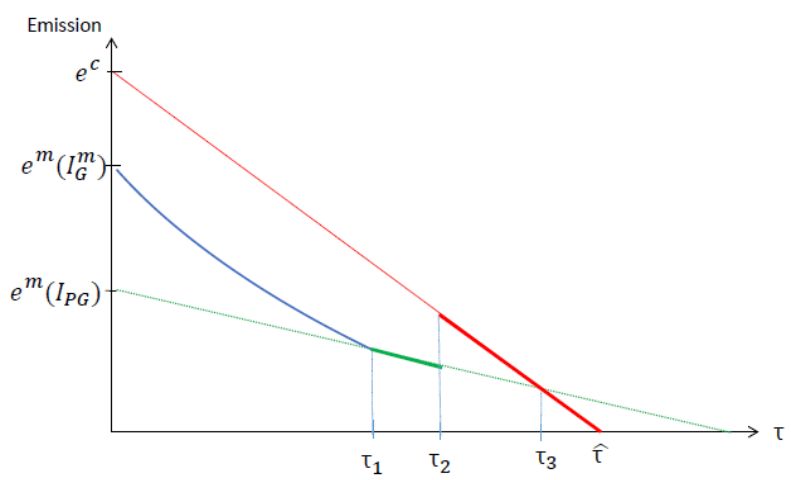

Figure 3: profit maximizing emission levels under weak patentability requirement

From Figure 3, it follows that, under a weak patentability requirement regime, for $\tau<\tau_{1}$, a sufficiently small increase in $\tau$ (a stronger environmental policy), reduces the emission level in equilibrium. A larger increase in $\tau$ to beyond $\tau_{1}$ pushes the profit-maximizing investment level down to $I_{P G}$ in which case, in order to be able to patent the firm cannot reduce its investment anymore and, thus, must invest at $I_{P G}$. At this investment level, as $\tau$ increases further, the emission level decreases but at a slower rate. At even higher values of $\tau$, i.e., beyond $\tau_{2}$, the

\footnotetext{
${ }^{26}$ Figure 3 illustrates the case for $\lambda \in\left(\lambda_{G}, 1\right]$. For $\lambda \in\left(\lambda_{1}, \lambda_{G}\right]$, at $\tau=0$ we have $e^{m}\left(I_{G}^{m}\right)<e^{m}\left(I_{P G}\right)$, since $I_{G}^{m}>I_{P G}$ and we are in Area II of Figure 2 whereby $\frac{\partial e^{m}\left(I_{G}\right)}{\partial I_{G}}<0$. As $\tau$ increases, $\lambda_{G}$ decreases such that we enter Area III of Figure 2 whereby $\frac{\partial e^{m}\left(I_{G}\right)}{\partial I_{G}}>0$. At the value of $\tau$ where this happens, $e^{m}\left(I_{G}^{m}\right) \operatorname{crosses} e^{m}\left(I_{P G}\right)$, and for higher values of $\tau$ we have $e^{m}\left(I_{G}^{m}\right)>e^{m}\left(I_{P G}\right)$. This does not qualitatively change our results.
} 
firm decides not to patent, and thus not to invest, in which case the emission level goes up to the competitive level, $e^{c}$ as shown in Figure 3. At a still higher tax rate, i.e., beyond $\tau_{3}, e^{c}$ falls below the emission level that would be reached if the innovation had occurred, $e^{m}\left(I_{P G}\right)$. Thus, unlike in the benchmark case with only non-green consumers the paradoxical result occurs only for an intermediate range of tax rates, $\tau \in\left(\tau_{2}, \tau_{3}\right)$. Holding constant $C_{P G}$, the paradox disappears by increasing tax rates beyond $\tau_{3}$ which reduces the emission level to $e^{c}$, despite the lack of innovation. At tax rates above $\tau_{3}$, we have $e^{m}\left(I_{P G}\right)>e^{c}$ for the following reason. While in the competitive equilibrium, there is no innovation, if $I_{P G}$ were invested, demand from the green consumers would be higher, pushing up the emission level.

Proposition 2 The emission level, in the presence of green consumers,

(i) decreases due to marginal increases in the tax rate at any $\tau \in\left[0, \tau_{2}[\right.$ and $\left.\tau \in] \tau_{2}, \widehat{\tau}\right]$,

(ii) increases discretely due to a marginal increase in the tax rate at $\tau_{2}$.

Proposition 2 generalizes the findings stated in Proposition 1 to the case with green consumers (see Appendix A for proof) with a caveat for very high tax rates. Comparing Proposition 1 and Proposition 2, it follows that for large emission tax levels, $\tau>\tau_{3}$, an increase in the emission tax level is only effective at reducing the emission level in the presence of green consumers.

We note that the threshold $\tau_{2}$ is decreasing in $C_{P G}$, by definition. ${ }^{27}$ This leads to the following Corollary.

Corollary 2 A sufficient reduction in the cost of patenting, $C_{P G}$,

(i) decreases the emission level for an intermediate range of tax rates, $\tau \in\left(\tau_{2}, \tau_{3}\right)$,

(ii) increases the emission level for very high tax rates, $\tau>\tau_{3}$.

Corollary 2 determines the conditions under which reducing patenting costs (e.g., by fasttracking green patents) helps to reduce emission levels, and when it does not. As per Corollary

\footnotetext{
${ }^{27}$ Recall that $C_{P G}=\left.\Pi^{m}\left(I_{P G}\right)\right|_{\tau=\tau_{2}}-I_{P G}$. Since $I_{P G}$ is constant and $\Pi^{m}\left(I_{P G}\right)$ is decreasing in $\tau$, we have that an increase in $C_{P G}$ must correspond to a decrease in $\tau_{2}$.
} 
$2(i)$, since the increase in emission occurs due to $\Pi^{m}\left(I_{G}^{m}\right)-I_{G}^{m}$ falling below $C_{P G}$ after an increase in $\tau$ beyond $\tau_{2}$, this adverse impact could be avoided by decreasing $C_{P G}$, as in the benchmark case without green consumers. However, in the presence of green consumers and for tax rates higher than $\tau_{3}$, as per Proposition $2(i i i)$, decreasing $C_{P G}$ increases the emission level from $e^{c}$ (which would occur without innovation if $C_{P G}$ were sufficiently high), to $e^{m}\left(I_{P G}\right)$, as shown in Figure 3.

The same results carry over to the case with a strong patentability requirement such that $I_{P G}>I_{G}^{m}$ for all $\tau \geq 0$, as per Definition 1. As shown in Figure 4, the emission level decreases as $\tau$ increases, and the same mechanism applies as described above, except that the firm is always constrained to invest $I_{P G}$. Note that the thresholds $\tau_{2}, \tau_{3}$ and $\widehat{\tau}$ are the same as in Figure 3. In Figure 4, the top (red) function represents $e^{c}$, the curved (dashed blue) one represents $e^{m}\left(I^{m}\right)$ when $I^{m}=I_{G}^{m}$ and the higher (green) one represents $e^{m}\left(I^{m}\right)$ when $I^{m}=I_{P G}$. The profit maximizing emission level is represented in bold. The paradoxical result occurs for the intermediate range of tax rates, $\tau \in\left(\tau_{2}, \tau_{3}\right) .{ }^{28}$

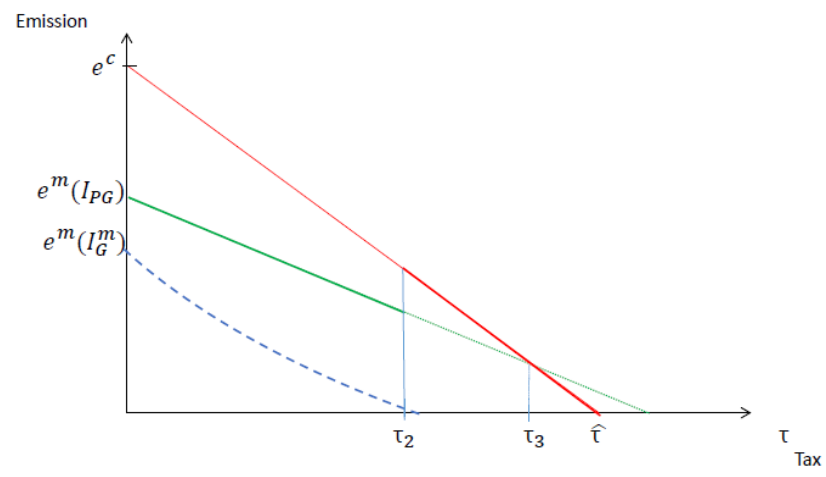

Figure 4: Profit maximizing emission levels with strong patent requirement

Next, we compare strict and weak patentability requirements in terms of their impact on emission levels.

\footnotetext{
${ }^{28}$ Figure 4 illustrates the case for $\lambda \in\left(\lambda_{G}, 1\right]$. For $\lambda \in\left(\lambda_{1}, \lambda_{G}\right]$, we have $e^{m}\left(I_{G}^{m}\right)>e^{m}\left(I_{P G}\right)$, since $I_{G}^{m}<I_{P G}$ and we are in Area II of Figure 2 whereby $\frac{\partial e^{m}\left(I_{G}\right)}{\partial I_{G}}<0$. As $\tau$ increases, $\lambda_{G}$ decreases such that we enter Area III of Figure 2 whereby $\frac{\partial e^{m}\left(I_{G}\right)}{\partial I_{G}}>0$. At the value of $\tau$ where this happens, $e^{m}\left(I_{G}^{m}\right)$ crosses $e^{m}\left(I_{P G}\right)$, and for higher values of $\tau$ we have $e^{m}\left(I_{G}^{m}\right)<e^{m}\left(I_{P G}\right)$. This does not qualitatively change our results.
} 
Proposition 3 The emission level is

(i) higher under a weak patentability requirement than under a strong patentability requirement for $\lambda \in\left(\lambda_{1}, \lambda_{G}\right]$;

(ii) lower under a weak patentability requirement than under a strong patentability requirement for $\lambda \in\left(\lambda_{G}, 1\right]$.

Proposition 3 follows directly from Lemma 1, and has direct and important policy implications. When the fraction of green conscious consumers is sufficiently small, i.e., $\lambda \in\left(\lambda_{1}, \lambda_{G}\right]$, moving from a weak to a strong patentability requirement for green innovation, ceteris paribus, decreases the emission level. This corresponds to Areas I and II in Figure 2. However, when the fraction of green conscious consumers is sufficiently large, i.e., $\lambda \in\left(\lambda_{G}, 1\right]$, moving from a weak to a strong patentability requirement for green innovation, ceteris paribus, increases the emission level, corresponding to Area III in Figure 2. Therefore, for $\lambda \in\left(\lambda_{G}, 1\right]$, the stricter the patentability requirement, that is, the higher that $I_{P G}$ is raised above $I_{G}^{m}$, the more urgent it becomes to increase the emission tax simultaneously in order to curb emissions.

\subsection{Socially Optimal Investment}

The socially optimal level of investment, given that the firm sets the monopoly price in the second period, is the solution of

$$
\underset{I_{G}}{\operatorname{Max}} W^{m}\left(I_{G}\right)=\Pi^{m}\left(I_{G}\right)-I_{G}+C S^{m}\left(I_{G}\right)+\tau e\left(I_{G}\right)-C_{P G},
$$

and is denoted by $I_{G}^{*} \cdot{ }^{29}$ The consumer surplus is given by:

$$
C S^{m}\left(I_{G}\right)=\frac{1}{2} \Pi^{m}\left(I_{G}\right)-e\left(I_{G}\right) .
$$

Thus, $I_{G}^{*}$ must satisfy $d W\left(I_{G}\right) / d I_{G}=0$ or, equivalently,

$$
\frac{d \Pi^{m}\left(I_{G}\right)}{d I_{G}}-1+\frac{d C S^{m}\left(I_{G}\right)}{d I_{G}}+\tau \frac{d e\left(I_{G}\right)}{d I_{G}}=0 .
$$

\footnotetext{
${ }^{29}$ We assume that taxes are a redistribution between consumers and producers. That is, taxes reduce profits and are re-distributed in a lump sum way to consumers. Since $\Pi^{m}\left(I_{G}\right)$, as defined by (10), denotes profits net of taxes, we add the tax revenue, $\tau e\left(I_{G}\right)$, back in the social welfare function, given by (15).
} 
Evaluated at $I_{G}^{m}$, the left-hand side of (16) above becomes

$$
\frac{1}{2}+(\tau-1) \frac{d e\left(I_{G}\right)}{d I_{G}}
$$

By Lemma 1, we have that $d e^{m}\left(I_{G}\right) / d I_{G}<(>) 0$ for $\lambda<(>) \lambda_{G}$. Thus, the expression (17) is positive for $\lambda>\lambda_{G}$ and $\tau>1$ and for $\lambda<\lambda_{G}$ and $\tau<1$. The expression (17) may be negative for $\lambda>\lambda_{G}$ and $\tau<1$ and for $\lambda<\lambda_{G}$ and $\tau>1$. There exists a threshold $\widetilde{\tau}_{1}>1$ such that for $\lambda<\lambda_{G}$ and $\tau>\widetilde{\tau}_{1}$, we have $(17)<0$. There exists a threshold $\widetilde{\tau}_{2}<1$ such that for $\lambda>\lambda_{G}$ and $\tau<\widetilde{\tau}_{2}$, we have $(17)<0$. This leads to the following Proposition.

Proposition 4 (i) For $\lambda \in\left(\lambda_{1}, \lambda_{G}\right]$ and $\tau \leq \widetilde{\tau}_{1}, I_{G}^{m}<I_{G}^{*}$ (the firm underinvests relative to the socially optimal level) and for $\tau>\widetilde{\tau}_{1}, I_{G}^{m}>I_{G}^{*}$ (the firm overinvests relative to the socially optimal level).

(ii) For $\lambda \in\left(\lambda_{G}, 1\right]$ and $\tau \geq \widetilde{\tau}_{2}, I_{G}^{m}<I_{G}^{*}$ (the firm underinvests relative to the socially optimal level) and for $\tau<\widetilde{\tau}_{2}, I_{G}^{m}>I_{G}^{*}$ (the firm overinvests relative to the socially optimal level).

Proposition 4(i) states that as long as the fraction of green conscious consumers is sufficiently low, the firm underinvests relative to the socially optimal level for a sufficiently low emission tax and overinvests for a sufficiently high emission tax. This is in line with much of the literature surrounding the Porter hypothesis which predicts that higher taxes induce more investment by firms. However, the gap between the socially efficient and privately optimal levels of investment steadily reduces as $\lambda$ increases, until this result is reversed when the fraction of green conscious consumers is sufficiently high, as stated by Proposition 4(ii).

\section{Duopoly}

We have thus far assumed that once a patent has been granted for a green technology that reduces the emission-output ratio below the threshold $\gamma_{P}$, the patented technology becomes a new technology standard for the industry which is enforced by regulators. This prevents other firms from producing the good with the old dirty technology, and allows us to focus on the monopoly case in order to clearly isolate and identify the direct channel through which 
consumers' behaviour impacts innovation and the emission level. In this section, we relax this assumption and consider a scenario where the new green technology can coexist with the old dirty technology in a duopoly game. We show that the paradoxical result whereby an increase in emission tax increases the emission level still holds for a range of parameter values even after allowing firms to behave strategically when setting prices.

In this section, we assume that Firm 1 produces a green product with an emission-output ratio given by $\gamma_{G}$ and price given by $p_{G}$. Firm 2 produces a dirty product with an emissionoutput ratio given by $\gamma_{H}$ and price given by $p_{H}$, where $\gamma_{H}>\gamma_{G}$ and $p_{H}<p_{G}$. That is, we allow Firm 1 to unilaterally invest in reducing $\gamma$. While all the details of the derivations of this duopoly model are provided in Appendix B, in this section, we summarize our main findings within this duopoly setting.

A green conscious consumer has the following utility function:

$$
U_{G}=\left\{\begin{array}{cc}
v-\gamma_{G} G-p_{G}-P(e) & \text { from buying the product from Firm } 1 \\
v-\gamma_{H} G-p_{H}-P(e) & \text { from buying the product from Firm } 2 \\
-P(e) & \text { from not buying }
\end{array}\right.
$$

A non-green conscious consumer always buys the dirty product since it is cheaper, and has the following utility function:

$$
U_{N G}=\left\{\begin{array}{cc}
v-p_{H}-P(e) & \text { from buying the product } \\
-P(e) & \text { from not buying }
\end{array}\right.
$$

A green conscious consumer $G$ buys the green product rather than the dirty product as long as the following condition holds:

$$
v-\gamma_{G} G-p_{G}-P(e)>v-\gamma_{H} G-p_{H}-P(e)
$$

that is, as long as $G \in\left(\frac{p_{G}-p_{H}}{\gamma_{H}-\gamma_{G}}, \bar{G}\right]$. Also, a green conscious consumer buys the green product rather than not buying anything as long as $v-\gamma_{G} G-p_{G}-P(e)>-P(e)$, that is, $G<$ $\left(p_{G}-v\right) / \gamma_{G}$. A non-green conscious consumer buys the dirty good as long as $v \geq p_{H}$. 
When both firms face positive demands, their demand functions are given by: ${ }^{30}$

$$
\begin{aligned}
D_{H} & =\lambda\left(\frac{p_{G}-p_{H}}{\gamma_{H}-\gamma_{G}}-\underline{G}\right)+(1-\lambda), \\
D_{G} & =\lambda\left(\frac{v-p_{G}}{\gamma_{G}}-\frac{p_{G}-p_{H}}{\gamma_{H}-\gamma_{G}}\right) .
\end{aligned}
$$

Within this setting, the impact of increasing investment by Firm 1 on the emission level is illustrated by Figure 5 .

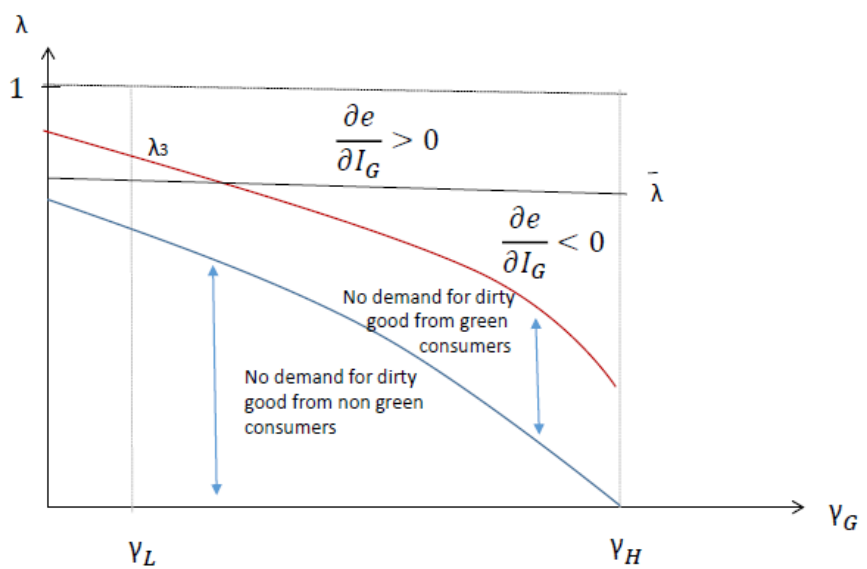

Figure 5: Impact of Increasing Investment in Green Technology on Emissions on Duopoly Case

Similarly to Figure 2, Figure 5 has an area (top area where $\lambda>\max \left\{\bar{\lambda}, \lambda_{3}\left(\gamma_{G}\right)\right\}$ ) where the emission level increases in the investment level in green innovation $\left(\partial e / \partial I_{G}>0\right)$. As the investment level increases, the emission of Firm 2 is reduced (as more consumers buy the cleaner product), but the emission of Firm 1 increases more than the decrease in the emission level of Firm 2. Thus, we illustrate that the key driving force behind the main results under monopoly carries over to the case of duopoly where a firm can strategically choose the greenness of its product.

Moreover, our results in terms of emission levels are qualitatively similar to those summarized by Figures 3 and 4 as long as $\gamma_{H}$ is sufficiently large. First, in the case of Bertrand competition (if Firm 1 does not innovate, and both firms produce the dirty product), we obtain the same equilibrium as in the monopoly case: the emission level is given by $e^{c}=\lambda\left(v-c-\gamma_{H}(\underline{G}+\tau)\right)+$ $\gamma_{H}(1-\lambda)$, which is decreasing with $\tau$. We define $\widehat{\tau}$ to be the value of the tax for which $e^{c}=0$, as

\footnotetext{
${ }^{30}$ In Appendix B, we provide the conditions on prices $p_{G}$ and $p_{H}$ such that both firms face positive demands.
} 
before. Then, we consider the case when the patenting constraint binds and Firm 1 must invest $I_{P G}\left(>I_{G}^{d}\right)$ in order to patent its innovation. In this case, the emission levels are, thus, given by $e_{G}\left(I_{P G}\right)=\gamma_{P} D_{G}(\tau)$ and $e_{H}\left(I_{P G}\right)=\gamma_{H} D_{H}(\tau)$, and both of them are decreasing with the $\operatorname{tax}, \tau$, as $\partial e_{G} / \partial \tau=\gamma_{P} \partial D_{G} / \partial \tau<0$ and $\partial e_{H} / \partial \tau=\gamma_{H} \partial D_{H} / \partial \tau<0$. Finally, we show that in the case where the patenting constraint is non-binding, as long as $\gamma_{H}>2 \gamma_{G}$, the total emission level is decreasing with the $\operatorname{tax}, \partial e(\tau) / \partial \tau<0$. Evaluated at $\tau=0, e\left(I_{G}^{d}\right)<e^{c}$, so that the emission levels in the case of our duopoly setting are similar to the monopoly case as represented in Figures 3 and 4. In particular, we retrieve the paradoxical result where an increase in $\tau$ leads to a increase in emission level for $\tau \in\left(\tau_{2}, \tau_{3}\right)$. However, in the duopoly case, whether $\tau_{3}$ is less that $\widehat{\tau}$ is ambiguous and depends on the values of $\gamma_{P}$ and $\gamma_{H}$.

\section{Conclusion}

In this paper, we develop a theoretical framework to investigate the impact of patent policies and emission taxes on green innovation and emissions in the presence of environmentally friendly consumers. We analyze the effect of changing patentability requirements and patenting costs when a firm may invest in a green innovation, which reduces the emission output ratio.

We show that, in the absence of green consumers, the introduction of patents may result in a paradoxical result whereby increasing the emission tax beyond a certain threshold leads to a discrete increase in the emission level, which may be avoided by reducing the patenting cost, e.g., by fast-tracking green patents. In the presence of green consumers, this paradox is restricted to an intermediate range of tax rates. This is because, at very high tax rates, the emission level in the competitive equilibrium that occurs in the absence of innovation, is lower than that with innovation. Thus, a lower emission level is reached despite a tax increase that makes the innovation unprofitable. It follows that, at sufficiently high tax rates, reducing patenting costs in order to induce innovation ends up increasing the emission level in the presence of green consumers.

Moreover, we show that a stricter patentability requirement is only effective at reducing emissions as long as the fraction of green consumers is sufficiently small. We also find that 
investment in green technologies reduces emissions only if the fraction of green consumers is sufficiently small, and that the magnitude of this effect decreases as the fraction of green consumers increases. If the fraction of green consumers increases beyond a certain threshold, investment in green technologies results in more emissions. To prevent this perverse outcome, policy makers might consider imposing a capacity constraint on firms as they invest more in green innovation in the presence of green consumers. For a sufficiently small fraction of green consumers, we retrieve the expected result found in much of the literature surrounding the Porter hypothesis that a higher emission tax increases green investment. However, this result is reversed if the fraction of green consumers rises to a level such that investment in green technologies results in more emissions. Finally, we show that as long as the fraction of green consumers is sufficiently low, the firm underinvests relative to the socially optimal level for a sufficiently low emission tax and overinvests for a sufficiently high emission tax. However, the gap between the socially efficient and privately optimal levels of investment steadily reduces as this fraction increases, until this result is reversed when the fraction of green consumers is sufficiently high.

To summarize, this paper determined the conditions under which reducing patenting costs and making patentability requirements stricter are effective at inducing green innovation and thereby reducing emissions. These patent policy tools were shown to become less effective as society becomes more environmentally friendly. Thus, further research seems warranted regarding the policies to reduce emissions through green innovation, and also regarding the type of information to distribute to consumers. In particular, if consumers reward marginal reductions in emission-ouput ratios of production processes by increasing their demand for the final product, this may inhibit the traditional patent policy tools from working as expected. At the same time, increasing emission taxes to very high levels seem more effective in the presence of green consumers, while increasing emission taxes may be harmful for the environment in the absence of green consumers. These are some of the policy implications generated in our framework.

As implied by the above summary of our findings, this paper generates a number of potentially testable hypotheses, each of which depends on the fraction of green consumers, $\lambda$. There are a few indices that measure the environmental friendliness of consumers across coun- 
tries, such as Greendex (computed by National Geographic) and GlobeScan, ${ }^{31}$ which may be used in conjunction with data on the relevant variables, such as emission and green investment levels and patenting costs, to test the hypotheses generated by the Lemmas and Propositions in this paper. Instead of using green consumer indices, another possible approach to testing our hypotheses might be the following two step procedure. First, the "rebound-like" effect as explained in Footnote 9, whereby the demand of a product increases the cleaner it becomes, would have to be estimated for different goods/regions. This would then act as a proxy for $\lambda$. Second, the interaction of this "rebound-like" effect with the relevant variables would need to be estimated. Testing the above hypotheses is beyond the scope of this paper, and we leave the relevant empirical analysis as future research.

The findings of this paper give rise to a number of questions which would be interesting to address in future work on this topic by extending the framework developed here. For example, in an open economy setting, where the degree of environmental friendliness of consumers is heterogeneous across countries, and polluting firms with market power are located across different countries, how are governments' strategies regarding the implementation of Intellectual Property Rights and fast-track patent systems affected? How do these policies affect the distribution of investment, pollution and welfare levels across countries? Also, in a general equilibrium setting, how does a drop in demand in the sector under consideration affect consumption in other sectors, and how does this affect total emissions?

\footnotetext{
${ }^{31}$ For further details refer to https://globescan.com/greendex-2014-consumer-choice-and-the-environment-aworldwide-tracking-survey-full-report/.
} 


\section{Appendix A}

\section{Figures}

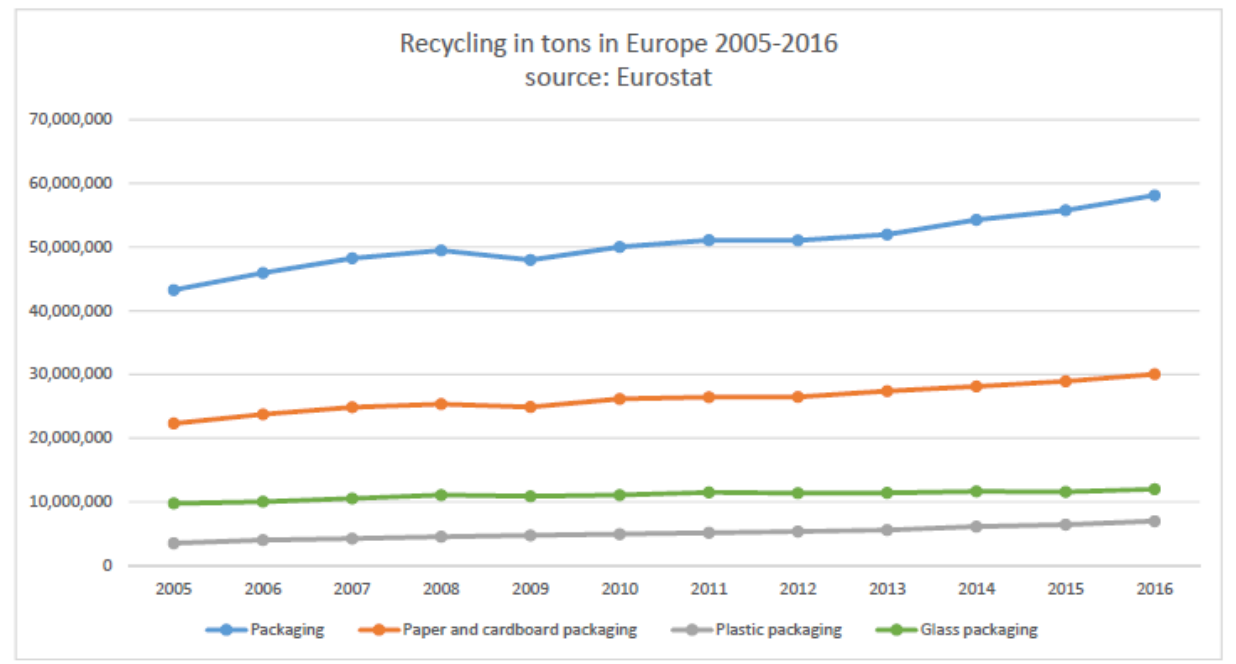

Figure A1

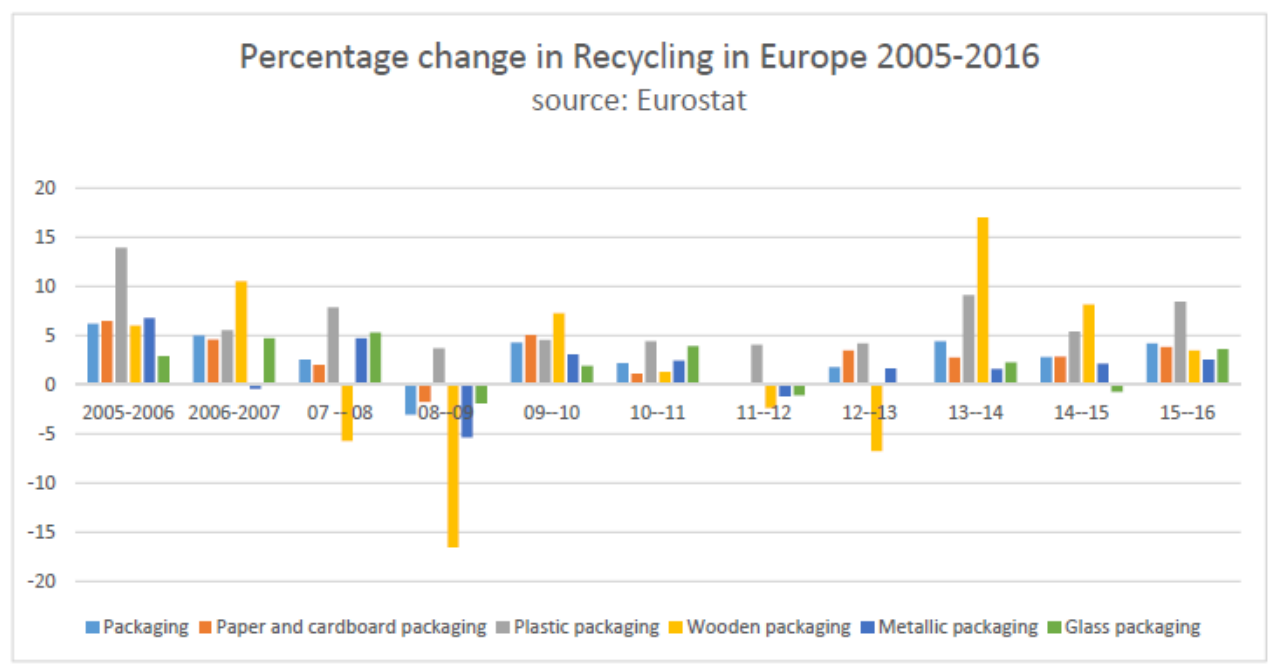

Figure A2

\section{Proof of Proposition 1}

The First Order Condition (FOC) in the first stage is given by:

$$
-\tau \gamma^{\prime}\left(I^{N G}\right)-1=0
$$


Let $f\left(\tau, I^{N G}\right) \equiv-\tau \gamma^{\prime}\left(I^{N G}\right)-1$. We then totally differentiate FOC $(20), f_{\tau} d \tau+f_{I} d I^{N G}=0$. Thus,

$$
\frac{d I^{N G}}{d \tau}=-\frac{f_{\tau}}{f_{I}}=-\frac{-\gamma^{\prime}\left(I^{N G}\right)}{-\tau \gamma^{\prime \prime}\left(I^{N G}\right)}>0
$$

Moreover,

$$
\frac{\partial e}{\partial \tau}=\frac{\partial \gamma}{\partial I} \frac{\partial I^{N G}}{\partial \tau}<0
$$

When $\tau=0$, we have $I^{N G}=0$ with $\gamma=\gamma_{H}$, with $\gamma$ decreasing in $\tau$ for $I^{N G}>0$. At $\tau=\hat{\tau}_{1}$, we have $\gamma\left(I^{N G}\right)=\gamma_{P}$. Thus, for a sufficiently low tax rate, that is, $\tau \in\left[0, \hat{\tau}_{1}\right)$, the firm invests the minimum amount required to obtain a patent, that is, $\gamma_{P}$. For $\tau_{1} \in\left[\hat{\tau}_{1}, \hat{\tau}_{2}\right)$, the firm has an incentive to invest more than this minimum amount since $\gamma\left(I^{N G}\right)<\gamma_{P}$. We note that the firm only invests as long as

$$
v-c-\tau \gamma\left(I^{N G}\right)-I^{N G}-C_{P G}>0
$$

which implies that

$$
v-c-C_{P G}>\tau \gamma\left(I^{N G}\right)+I^{N G},
$$

with $\frac{\partial}{\partial \tau}\left(\tau \gamma\left(I^{N G}\right)+I^{N G}\right)=\gamma\left(I^{N G}\right)+\frac{\partial I}{\partial \tau}\left(\tau \frac{\partial \gamma}{\partial I}+1\right)$. By $(20)$, we have that $\left(\tau \frac{\partial \gamma}{\partial I}+1\right)=0$ such that $\frac{\partial}{\partial \tau}\left(\tau \gamma\left(I^{N G}\right)+I^{N G}\right)>0$. Thus, there exists a threshold $\hat{\tau}_{2}$ such that for $\tau \in\left[0, \hat{\tau}_{2}\right)$, we have $v-c-C_{P G}>\tau \gamma\left(I^{N G}\right)+I^{N G}$ and for $\tau>\hat{\tau}_{2}$, we have $v-c-C_{P G} \leq \tau \gamma\left(I^{N G}\right)+I^{N G}$. It follows that for $\tau>\hat{\tau}_{2}$, the firm does not invest and Bertrand competition results in emission level given by $e^{c}=\gamma_{H}$. 


\section{Monopoly Pricing}

The firm chooses $p^{m}$ as defined by (8) instead of $v$ if the profit (10) is higher than the profit (7), i.e., $\Pi^{m}>\Pi(v)$ or, equivalently,

$$
\begin{aligned}
\frac{\lambda}{4 \gamma}\left[v-c-\gamma(\underline{G}+\tau)+\frac{1-\lambda}{\lambda} \gamma\right]^{2} & >(v-c-\gamma \tau)(1-\lambda), \\
{\left[v-c-\gamma \tau-\gamma \underline{G}+\frac{1-\lambda}{\lambda} \gamma\right]^{2} } & >4 \gamma \frac{1-\lambda}{\lambda}(v-c-\gamma \tau) .
\end{aligned}
$$

Let us denote $a=v-c-\gamma \tau$ and $b=\frac{1-\lambda}{\lambda} \gamma$. The inequality can be rewritten as $[a-\gamma \underline{G}+b]^{2}>4 b a$ or

$$
b^{2}-2 b(a+\gamma \underline{G})+(a-\gamma \underline{G})^{2}>0 .
$$

Thus, the discriminant of the quadratic equation is $\Delta=4(a+\gamma \underline{G})^{2}-4(a-\gamma \underline{G})^{2}=16 a \gamma \underline{G}>0$, and the two roots are

$$
\begin{aligned}
b^{\prime} & =(a+\gamma \underline{G})-2 \sqrt{a \gamma \underline{G}}>0, \\
b^{\prime \prime} & =(a+\gamma \underline{G})+2 \sqrt{a \gamma \underline{G}} .
\end{aligned}
$$

Therefore, for values of $b$ such that $b<b^{\prime}$ and $b>b^{\prime \prime}$ inequality (22) is satisfied. In terms of $\lambda$, we obtain that this inequality is satisfied as long as $\lambda>\lambda_{1}$ and $\lambda<\lambda_{2}$ with

$$
\begin{aligned}
& \lambda_{1}=\frac{\gamma}{\gamma+v-c+\gamma(\underline{G}-\tau)-2(v-c-\gamma \tau)^{\frac{1}{2}}(\gamma \underline{G})^{\frac{1}{2}}}, \\
& \lambda_{2}=\frac{\gamma}{\gamma+v-c+\gamma(\underline{G}-\tau)+2(v-c-\gamma \tau)^{\frac{1}{2}}(\gamma \underline{G})^{\frac{1}{2}}} .
\end{aligned}
$$

We show that $\lambda_{2}<\underline{\lambda}$, so that the firm will choose the monopoly price (8) if $\lambda>\lambda_{1}$.

\section{Proof of Lemma 1}

From (12), we have $\frac{\partial e^{m}\left(I_{G}\right)}{\partial \tau}=-\frac{\lambda \gamma}{2}<0$, which results in Lemma $1(i)$. The derivative of $e^{m}\left(I_{G}\right)$ with respect to $I_{G}$ is given by:

$$
\frac{\partial e^{m}\left(I_{G}\right)}{\partial I_{G}}=\frac{1}{2}(1-\lambda(\underline{G}+\tau+1)) \frac{d \gamma}{d I_{G}},
$$

where $d \gamma / d I_{G}<0$. Thus, $\partial e^{m}\left(I_{G}\right) / \partial I_{G}<(>) 0$ for $\lambda<(>) \lambda_{G}$, which results in Lemma $1(i i)$. 


\section{Second Order Condition}

In the first period, the firm chooses the optimal investment that satisfies

$$
\left\{\begin{array}{l}
\underset{I_{G}}{\operatorname{Max} \Pi^{m}}\left(I_{G}\right)-I_{G} \\
\text { s.t. } \gamma\left(I_{G}\right) \leq \gamma_{P}
\end{array}\right.
$$

where the profit $\Pi^{m}\left(I_{G}\right)$ is defined by (10). Let $I_{G}^{m}$ be the solution of the unconstrained optimization program (i.e., $I_{G}^{m}=\arg \max \Pi^{m}\left(I_{G}\right)-I_{G}$ ) such that it is solution of (13). Notice that the expression

$$
\frac{\lambda}{4}\left(\left(\frac{v-c}{\gamma}\right)^{2}-(\underline{G}+\tau)^{2}\right)+\frac{(1-\lambda)}{2}(\underline{G}+\tau)-\frac{1}{4} \frac{(1-\lambda)^{2}}{\lambda}
$$

is strictly concave for all $0<\lambda<1$, with two roots given by $\tilde{\lambda}=\frac{\gamma}{v-c+\gamma+G \gamma+\tau \gamma}$ and $\bar{\lambda}=$ $\frac{\gamma}{c-v+\gamma+G \gamma+\tau \gamma}>1$. It can be shown that $\tilde{\lambda}<\underline{\lambda}<\lambda_{G}<1<\bar{\lambda}$. Therefore, the left-hand side of equation (13) is positive such that (13) is satisfied.

The Second Order Condition (SOC) is satisfied as long as

$$
\frac{\lambda}{2}\left(\gamma^{\prime}\right)^{2} \frac{(v-c)^{2}}{\gamma^{3}}-\gamma^{\prime \prime}\left(\frac{\lambda}{4}\left(\left(\frac{v-c}{\gamma}\right)^{2}-(\underline{G}+\tau)^{2}\right)+(1-\lambda)(\underline{G}+\tau)-\frac{1}{4} \frac{(1-\lambda)^{2}}{\lambda}\right)<0 .
$$

Using the First Order Condition (13) we can write

$$
-\left(\frac{\lambda}{4}\left(\left(\frac{v-c}{\gamma}\right)^{2}-(\underline{G}+\tau)^{2}\right)+(1-\lambda)(\underline{G}+\tau)-\frac{1}{4} \frac{(1-\lambda)^{2}}{\lambda}\right)=\frac{1}{\gamma^{\prime}\left(I_{G}\right)},
$$

that we plug into the SOC to obtain a local condition

$$
\frac{\lambda}{2}\left(\gamma^{\prime}\right)^{2} \frac{(v-c)^{2}}{\gamma^{3}}+\frac{\gamma^{\prime \prime}}{\gamma^{\prime}\left(I_{G}\right)}<0
$$

or

$$
\gamma^{\prime \prime}(.)>-\lambda \frac{(v-c)^{2}}{2}\left(\frac{\gamma^{\prime}(.)}{\gamma(.)}\right)^{3}
$$

which is Assumption (A3). If this local condition is satisfied, locally the profit function is concave.

\section{Comparative Statics}

Lemma 5 For a weak patentability requirement, $I_{G}^{m}$ is decreasing in $c$, and increasing in $v$.

Lemma 5 follows directly from (13) (see below for details of the calculations). As the marginal cost of production, $c$, increases, the profitability of the product decreases, which explains why 
firms have less incentive to invest in the green technology. As $v$ increases, demand increases, leading to an increase in $I_{G}^{m}$.

\section{Proofs of Lemmas 2 and 5}

In order to derive the relevant comparative statics results on $I_{G}^{m}$, it is useful to define the following:

$$
F\left(I_{G}, y\right) \equiv-\gamma^{\prime}\left(\frac{\lambda}{4}\left(\left(\frac{v-c}{\gamma}\right)^{2}-(\underline{G}+\tau)^{2}\right)+\frac{(1-\lambda)}{2}(\underline{G}+\tau)-\frac{1}{4} \frac{(1-\lambda)^{2}}{\lambda}\right)-1
$$

where $y$ may represent any of the exogenously given parameters, that is, $y \in\{\tau, c, v, \underline{G}, \lambda\}$. By totally differentiating (13), we have the following

$$
\frac{d I_{G}^{m}}{d y}=-\frac{\frac{\partial F}{\partial y}}{\frac{\partial F}{\partial I_{G}}} .
$$

Since we have assumed the existence of an interior solution, it follows that a maximum is reached at $I_{G}^{m}$. This implies that $\frac{\partial F}{\partial I_{G}} \leq 0$, and thus

$$
\operatorname{sign} \frac{d I_{G}^{m}}{d y}=\operatorname{sign} \frac{\partial F}{\partial y} \text {. }
$$

For $y \in\{\tau, c, v, \underline{G}, \lambda\}$, we have

$$
\frac{\partial F}{\partial \tau}=\frac{\partial F}{\partial \underline{G}}=\frac{\gamma^{\prime}}{2}(\lambda(\tau+\underline{G})-(1-\lambda))<0
$$

if and only if $\lambda \geq \lambda_{G}$.

$$
\begin{gathered}
\frac{\partial F}{\partial c}=\lambda \frac{\gamma^{\prime}}{2} \frac{v-c}{\gamma^{2}}<0, \\
\frac{\partial F}{\partial v}=-\lambda \frac{\gamma^{\prime}}{2} \frac{v-c}{\gamma^{2}}>0 .
\end{gathered}
$$

The following table summarizes the above comparative statics results.

\begin{tabular}{|l|l||}
\hline \multicolumn{2}{|c||}{ TABLE 1: Comparative Statics } \\
\hline Parameter $y$ & $\operatorname{sign}\left(\frac{d I_{G}^{m}}{d y}\right)$ \\
\hline$\tau$ & - \\
\hline$c$ & - \\
\hline$v$ & + \\
\hline$\underline{G}$ & - \\
\hline
\end{tabular}




\section{Figure 3}

The emission level when the price is equal to marginal cost is given by equation (14), $e^{c}=$ $\lambda\left(v-c-\gamma_{H}(\underline{G}+\tau)\right)+\gamma_{H}(1-\lambda)$ which is decreasing with $\tau$ at the constant rate $-\lambda \gamma_{H}$. The emission level is positive for $\tau<\widehat{\tau} \equiv(v-c) / \gamma_{H}-\underline{G}+(1-\lambda) / \lambda$, which is satisfied according to Assumption (A1). It is represented by the top (red) function. The bottom (green) function represents the emission level when the patenting constraint is binding $e^{m}\left(I_{P G}\right)=\lambda\left(v-c-\gamma_{P}(\underline{G}+\right.$ $\tau)) / 2+(1-\lambda) \gamma_{P} / 2$. This function is also decreasing with $\tau$ at the constant rate $-\lambda \gamma_{P} / 2>-\lambda \gamma_{H}$. The emission level is positive for $\tau<(v-c) / \gamma_{P}-\underline{G}+(1-\lambda) / \lambda$ where $(v-c) / \gamma_{P}-\underline{G}+(1-\lambda) / \lambda>\widehat{\tau}$. The two functions intersect at $\tau=(v-c) /\left(2 \gamma_{H}-\gamma_{P}\right)-\underline{G}+(1-\lambda) / \lambda$. The middle (blue) curve represents the emission level at the profit-maximizing investment level $I_{G}^{m}$, and it intersects with $e^{m}\left(I_{P G}\right)$ at $\widetilde{\tau}$ which is where $\gamma\left(I_{G}^{m}\right)=\gamma_{P}$ or $I_{G}^{m}=I_{P G}$. We also show that, evaluated at $\tau=0, e^{m}\left(I_{P G}\right)<e^{c}$ is satisfied if $\gamma_{H}<(v-c) / 2 \underline{G}$ and we have $e^{m}\left(I_{G}^{m}\right)>e^{m}\left(I_{P G}\right)$ as long as $\lambda>1 /(\underline{G}+1)$.

\section{Proof of Lemma 4}

As long as $I_{P G} \leq I_{G}^{m}$, i.e., the patentability requirement is weak, and $C_{P G}<\Pi^{m}\left(I_{G}^{m}\right)-I_{G}^{m}$, it is profitable to invest. If $I_{P G}>I_{G}^{m}$ for all $\tau$, i.e., the patentability requirement is strong, then unless $C_{P G}<\Pi^{m}\left(I_{P G}\right)-I_{P G}<\Pi^{m}\left(I_{G}^{m}\right)-I_{G}^{m}$, it is not profitable to invest.

\section{Proof of Proposition 2}

The emission level, $e^{m}\left(I^{m}\right)$, is decreasing in $\tau$ since

$$
\frac{\partial e^{m}\left(I^{m}\right)}{\partial \tau}=\frac{d e^{m}}{d \tau}+\frac{\partial e^{m}\left(I^{m}\right)}{\partial I^{m}} \frac{\partial I^{m}}{\partial \tau}<0 .
$$

For any $\tau_{2}>\tilde{\tau}$, the relevant emission level is $e^{m}\left(I_{P G}\right)$ since we have that $I_{P G}>I_{G}^{m}$ for this range of $\tau$. If $I^{m}=I_{P G}$, the second term of (23) is null, and we have that $d e^{m} / d \tau=-\lambda \gamma\left(I^{m}\right) / 2<0$. For any $\tau_{1}<\tilde{\tau}$, the relevant emission level is $e^{m}\left(I_{G}^{m}\right)$ since we have that $I_{P G} \leq I_{G}^{m}$ for this range of $\tau$. If $I^{m}=I_{G}^{m}$, the second term of (23) is also negative since $\partial e^{m} / \partial I^{m}<(>) 0$ for $\lambda<(>) \lambda_{G}$ by Lemma 1 , and $\partial I^{m} / \partial \tau>(<) 0$ for $\lambda<(>) \lambda_{G}$ by Lemma 2 . It follows that $\left|\frac{\partial e^{m}\left(I_{G}^{m}\right)}{\partial \tau}\right|>\left|\frac{\partial e^{m}\left(I_{P G}\right)}{\partial \tau}\right|$. This completes the proof of Proposition 3(i). An increase in $\tau$ from either $\tau_{1}$ or $\tau_{2}$ to $\tau_{3}$ causes $\Pi^{m}\left(I_{G}^{m}\right)-I_{G}^{m}$ to fall below $C_{P G}$ such that it becomes unprofitable 
for the firm to invest in the green technology, as per Proposition 2. This causes the emission level to rise from $e^{m}\left(I^{m}\right)$ to $e^{c}$ as given by (14). This completes the proof of Proposition 3(ii).

\section{Socially Optimal Investment}

The total welfare is

$$
W^{m}\left(I_{G}\right)=\Pi^{m}\left(I_{G}\right)-I_{G}-C_{P G}+\tau e+C S^{m}\left(I_{G}\right)
$$

where $\Pi^{m}\left(I_{G}\right)$ is defined by (10) and the consumer surplus is

$$
\begin{aligned}
C S^{m}\left(I_{G}\right) & =\lambda \int_{\underline{G}}^{\frac{v-p}{\gamma}}(v-\gamma G-p-P(e)) d F+\lambda \int_{\frac{v-p}{\gamma}}^{\bar{G}}(-P(e)) d F+(1-\lambda)(-P(e)) \\
& =\frac{\lambda}{8 \gamma}\left(v-c-\gamma(\underline{G}+\tau)-\frac{1-\lambda}{\lambda} \gamma\right)^{2}-e .
\end{aligned}
$$

Therefore, the total welfare can be written as

$$
W^{m}\left(I_{G}\right)=\frac{3}{2}\left(\Pi^{m}\left(I_{G}\right)-I_{G}\right)+\frac{1}{2} I_{G}-(1-\tau) e-C_{P G} .
$$

The derivative of the total welfare gives

$$
\frac{d W^{m}\left(I_{G}\right)}{d I_{G}}=\frac{3}{2}\left(\frac{d \Pi^{m}\left(I_{G}\right)}{d I_{G}}-1\right)+\frac{1}{2}-(1-\tau) \frac{d e(.)}{d I_{G}} .
$$

Evaluated at $I_{G}^{m}$, it becomes expression (14) or

$$
\frac{1}{2}+(\tau-1) \frac{d e(.)}{d I_{G}}
$$

It is positive for $\lambda>\lambda_{G}$ and $\tau>1$ and for $\lambda<\lambda_{G}$ and $\tau<1$. There exists a threshold $\widetilde{\tau}_{1}>1$ such that for $\lambda<\lambda_{G}$ and $\tau>\widetilde{\tau}_{1}$, we have $(17)<0$. There exists a threshold $\widetilde{\tau}_{2}<1$ such that for $\lambda>\lambda_{G}$ and $\tau<\widetilde{\tau}_{2}$, we have $(17)<0$. 


\section{Appendix B - Duopoly Case}

Consider a duopoly case in which one of the firms, Firm 1, gets a patent for the green technology it has discovered, while the other firm, Firm 2, still produces its dirty product. Firm 1 produces a green product, the emission-output ratio of which is given by $\gamma\left(I_{G}\right)=\gamma_{G}$ at price $p_{G}$, and Firm 2 produces the dirty product, the emission-output ratio of which is given by $\gamma_{H}$ at price $p_{H}$, where $\gamma_{G}<\gamma_{H}$. Let's assume $p_{G}>p_{H}$.

The timing is similar to the monopoly case: in the first stage of the game, Firm 1 decides the level of investment in the green technology, $I_{G}$. Once the innovation has been discovered, Firm 1 decides whether to patent it. In the second stage, both firms choose their prices simultaneously. The patent prevents Firm 2 from producing with the cleaner technology and, thus, Firm 2 can only use the dirty technology.

Unlike the monopoly case, there are now two demand functions: one for the green product and one for the dirty product. A green conscious consumer has the following utility function:

$$
U_{G}=\left\{\begin{array}{cc}
v-\gamma_{G} G-p_{G}-P(e) & \text { from buying the product from Firm } 1 \\
v-\gamma_{H} G-p_{H}-P(e) & \text { from buying the product from Firm } 2 \\
-P(e) & \text { from not buying }
\end{array}\right.
$$

A non-green conscious consumer has the following utility function:

$$
U_{N G}=\left\{\begin{array}{cc}
v-p_{H}-P(e) & \text { from buying the product } \\
-P(e) & \text { from not buying }
\end{array}\right.
$$

A green conscious consumer $G$ buys the green product rather than the dirty product as long as $v-\gamma_{G} G-p_{G}-P(e)>v-\gamma_{H} G-p_{H}-P(e)$, that is, as long as $G \in\left(\frac{p_{G}-p_{H}}{\gamma_{H}-\gamma_{G}}, \bar{G}\right]$. Also, a green conscious consumer buys the green product rather than not buying anything as long as $v-\gamma_{G} G-p_{G}-P(e)>-P(e)$, that is, $G<\left(p_{G}-v\right) / \gamma_{G}$. A non-green consumer buys the dirty good as long as $v \geq p_{H}$. 
The demand for the dirty product is, thus, given by:

$$
D_{H}=\left\{\begin{array}{lll}
1 & \text { if } & p_{H} \leq v<\frac{\gamma_{G}}{\gamma_{H}} p_{H}+v \frac{\gamma_{H}-\gamma_{G}}{\gamma_{H}}<p_{G} \\
\lambda\left(\frac{p_{G}-p_{H}}{\gamma_{H}-\gamma_{G}}-\underline{G}\right)+(1-\lambda) & \text { if } & p_{H} \leq v<p_{H}+\left(\gamma_{H}-\gamma_{G}\right) \underline{G}<p_{G}<\frac{\gamma_{G}}{\gamma_{H}} p_{H}+v \frac{\gamma_{H}-\gamma_{G}}{\gamma_{H}} \\
(1-\lambda) & \text { if } & p_{H} \leq v<p_{G}<p_{H}+\left(\gamma_{H}-\gamma_{G}\right) \underline{G} \\
0 & \text { if } & v<p_{H}<p_{G}
\end{array}\right.
$$

The demand for the green product is given by:

$$
D_{G}=\left\{\begin{array}{lll}
\lambda & \text { if } \quad p_{H} \leq v<p_{G}<p_{H}+\left(\gamma_{H}-\gamma_{G}\right) \underline{G} \\
\lambda\left(\frac{v-p_{G}}{\gamma_{G}}-\frac{p_{G}-p_{H}}{\gamma_{H}-\gamma_{G}}\right) & \text { if } \quad p_{H} \leq v<p_{H}+\left(\gamma_{H}-\gamma_{G}\right) \underline{G}<p_{G}<\frac{\gamma_{G}}{\gamma_{H}} p_{H}+v \frac{\gamma_{H}-\gamma_{G}}{\gamma_{H}} \\
0 & \text { if } \quad p_{H} \leq v<\frac{\gamma_{G}}{\gamma_{H}} p_{H}+v \frac{\underline{\gamma}_{H}-\gamma_{G}}{\gamma_{H}}<p_{G}
\end{array}\right.
$$

\section{Green Conscious Consumers - Duopoly Pricing}

Given that Firm 1 has made an innovation and has patented it, we determine the equilibrium prices for a given investment level $I_{G}$.

First, we show that Firm 2 will not set its price $p_{H}=v$ as the condition $v<p_{G}$ will not be satisfied. Thus, Firm 2 must set its price at $p_{H}<v$. When the firms serve both types of consumers, Firm 1 will choose $p_{G}$ that solves the following:

$$
\operatorname{Max}\left(p_{G}-c-\tau \gamma_{G}\right) \lambda\left(\frac{v}{\gamma_{G}}+\frac{p_{H}}{\gamma_{H}-\gamma_{G}}-p_{G} \frac{\gamma_{H}}{\gamma_{G}\left(\gamma_{H}-\gamma_{G}\right)}\right) .
$$

We obtain the best response function of Firm 1 as a function of the price of the dirty product, as follows:

$$
p_{G}\left(p_{H}\right)=\frac{1}{2}\left(\frac{\gamma_{G}}{\gamma_{H}} p_{H}+\frac{v}{\gamma_{H}}\left(\gamma_{H}-\gamma_{G}\right)+c+\tau \gamma_{G}\right) .
$$

Firm 2 chooses $p_{H}$ that solves the following:

$$
\operatorname{Max}\left(p_{H}-c-\tau \gamma_{H}\right)\left(\lambda\left(\frac{p_{G}-p_{H}}{\gamma_{H}-\gamma_{G}}-\underline{G}\right)+(1-\lambda)\right),
$$

which gives the following best response function:

$$
p_{H}\left(p_{G}\right)=\frac{1}{2}\left(p_{G}+\left(\frac{1-\lambda}{\lambda}-\underline{G}\right)\left(\gamma_{H}-\gamma_{G}\right)+c+\tau \gamma_{H}\right) \text {. }
$$

Solving the two best response functions simultaneously, we obtain the following profit-maximizing prices:

$$
\begin{aligned}
& p_{G}^{*}=\frac{1}{4 \gamma_{H}-\gamma_{G}}\left(\left(\gamma_{H}-\gamma_{G}\right)\left(\gamma_{G}\left(\frac{1-\lambda}{\lambda}-\underline{G}\right)+2 v\right)+\left(\gamma_{G}+2 \gamma_{H}\right) c+3 \tau \gamma_{H} \gamma_{G}\right), \\
& p_{H}^{*}=\frac{1}{4 \gamma_{H}-\gamma_{G}}\left(\left(\gamma_{H}-\gamma_{G}\right)\left(2 \gamma_{H}\left(\frac{1-\lambda}{\lambda}-\underline{G}\right)+v\right)+\left(\gamma_{G}+2 \gamma_{H}\right) \tau \gamma_{H}+3 \gamma_{H} c\right) .
\end{aligned}
$$


We verify that $p_{G}^{*}>p_{H}^{*}$ if $v-c-2 \tau \gamma_{H}+\bar{G}\left(2 \gamma_{H}-\gamma_{G}\right)>0$. We also check that $p_{H}^{*}<v$ if $\lambda>\lambda_{1}\left(\gamma_{G}\right)$, where

$$
\lambda_{1}\left(\gamma_{G}\right) \equiv \frac{2\left(\gamma_{H}-\gamma_{G}\right)}{3\left(v-c-\gamma_{G} \tau\right)+(2 \underline{G}+1-2 \tau)\left(\gamma_{H}-\gamma_{G}\right)} .
$$

To obtain positive demands for both products, we check that $v-\left(\gamma_{H}-\gamma_{G}\right) \underline{G}<p_{H}^{*}$ if $\lambda<\lambda_{2}\left(\gamma_{G}\right)$, where

$$
\lambda_{2}\left(\gamma_{G}\right) \equiv \frac{2\left(\gamma_{H}-\gamma_{G}\right)}{\left.(v-c)+\left(2 \gamma_{H}+\gamma_{G}\right)(\underline{G}-\tau)+2\left(\gamma_{H}-\gamma_{G}\right)+2 \gamma_{G} \underline{G}+\gamma_{G} \frac{\gamma_{G} G}{\gamma_{H}}\right)},
$$

and that $\left(\gamma_{H}-\gamma_{G}\right) \underline{G}<p_{G}^{*}-p_{H}^{*}$ if $\lambda>\lambda_{3}\left(\gamma_{G}\right)$, where

$$
\lambda_{3}\left(\gamma_{G}\right) \equiv \frac{2 \gamma_{H}-\gamma_{G}}{v-c-2 \gamma_{H}(\tau+\underline{G})+2 \gamma_{H}-\gamma_{G}} .
$$

Lastly, we need to check that $p_{G}^{*}<\frac{\gamma_{G}}{\gamma_{H}} p_{H}^{*}+v \frac{\gamma_{H}-\gamma_{G}}{\gamma_{H}}$ since $\lambda<1$.

Both demands evaluated at prices $\left(p_{G}^{*}, p_{H}^{*}\right)$ are, thus, given by:

$$
D_{H}\left(p_{G}^{*}, p_{H}^{*}\right)=\frac{1}{4 \gamma_{H}-\gamma_{G}}\left(\lambda\left(v-c-2 \gamma_{H}(\tau+1+\underline{G})\right)+2 \gamma_{H}\right)
$$

and

$$
D_{G}\left(p_{G}^{*}, p_{H}^{*}\right)=\frac{\gamma_{H}}{\gamma_{G}\left(4 \gamma_{H}-\gamma_{G}\right)}\left(\lambda\left(2(v-c)-\gamma_{G}(\underline{G}+\tau+1)\right)+\gamma_{G}\right) .
$$

The demand for the dirty product decreases with the investment level in green technology, $I_{G}$, as

$$
\frac{\partial D_{H}\left(I_{G}\right)}{\partial I_{G}}=\lambda \gamma_{G}^{\prime}\left(I_{G}\right) \frac{v-c-2\left(\underline{G}+\tau-\frac{1-\lambda}{\lambda}\right) \gamma_{H}}{\left(4 \gamma_{H}-\gamma_{G}\right)^{2}}<0 .
$$

However, the demand for the green good decreases with the investment level, $I_{G}$, only if $\lambda<$ $\lambda_{5}\left(\gamma_{G}\right)$, but increases with the investment level for $\lambda>\lambda_{5}\left(\gamma_{G}\right)$, where

$$
\lambda_{5}\left(\gamma_{G}\right) \equiv \frac{\gamma_{G}^{2}}{\gamma_{G}^{2}(\underline{G}+\tau+1)+4(v-c)\left(2 \gamma_{H}-\gamma_{G}\right)},
$$

where $\lambda_{5}\left(\gamma_{G}\right)<\lambda_{4}\left(I_{G}\right)$. The response of demand to marginal changes in investment is given by:

$$
\frac{\partial D_{G}\left(I_{G}\right)}{\partial I_{G}}=\gamma_{H} \gamma_{G}^{\prime}\left(I_{G}\right) \frac{\gamma_{G}^{2}-\lambda \gamma_{G}^{2}(\underline{G}+\tau+1)-4 \lambda(v-c)\left(2 \gamma_{H}-\gamma_{G}\right)}{\gamma_{G}^{2}\left(4 \gamma_{H}-\gamma_{G}\right)^{2}} .
$$

If the fraction of green conscious consumers, $\lambda$, is large enough, an increase in the investment level in green technology increases the demand for the green product. 
The emission from the dirty producer is given by $e_{H}=\gamma_{H} D_{H}\left(p_{G}^{*}, p_{H}^{*}\right)$, which is always decreasing with the investment level, $I_{G}$, as

$$
\frac{\partial e_{H}}{\partial I_{G}}=\gamma_{H} \lambda \gamma_{G}^{\prime}\left(I_{G}\right) \frac{v-c-2\left(\underline{G}+\tau-\frac{1-\lambda}{\lambda}\right) \gamma_{H}}{\left(4 \gamma_{H}-\gamma_{G}\left(I_{G}\right)\right)^{2}}<0
$$

As Firm 1 invests in cleaner technology, the emission of the dirty product will be reduced as the demand decreases.

The emission from the green producer is given by $e_{G}=\gamma_{G}\left(I_{G}\right) D_{G}\left(I_{G}\right)$. As the investment level increases, the emission level increases if $\lambda>\bar{\lambda}$ where

$$
\bar{\lambda} \equiv \frac{2 \gamma_{H}}{2(\underline{G}+\tau+1) \gamma_{H}-(v-c)},
$$

as

$$
\frac{\partial e_{G}}{\partial I_{G}}=\gamma_{G}^{\prime}\left(I_{G}\right) \gamma_{H} \frac{2}{\left(4 \gamma_{H}-\gamma_{G}\right)^{2}}\left(\lambda(v-c)-\lambda(\underline{G}+\tau+1) 2 \gamma_{H}+2 \gamma_{H}\right) .
$$

We can also derive the total emission level given by $e=e_{H}+e_{G}=\gamma_{H} D_{H}+\gamma_{G}(I) D_{G}(I)$, which is decreasing in $\tau$ as

$$
\frac{\partial e}{\partial \tau}=\gamma_{H} \frac{-\lambda\left(2 \gamma_{H}+\gamma_{G}(I)\right)}{4 \gamma_{H}-\gamma_{G}(I)}<0
$$

For a given investment level, as $\tau$ increases, the total emission level decreases. However, when we account for the profit-maximizing investment level, the effect of an increase of investment on the emission level can be decomposed as follows:

$$
\frac{\partial e}{\partial I_{G}}=\underbrace{\frac{\partial e_{H}}{\partial I_{G}}}_{(-)}+\underbrace{\frac{\partial e_{G}}{\partial I_{G}}}_{(+ \text {or }-)} .
$$

Thus, an increase in the investment level decreases the emission level of Firm 2, but may increase or decrease the emission level of Firm 1. Formally, we have that

$$
\frac{\partial e}{\partial I_{G}}=\gamma_{G}^{\prime} \frac{3 \gamma_{H}}{\left(4 \gamma_{H}-\gamma_{G}\right)^{2}}\left(\lambda\left((v-c)-2(\underline{G}+\tau+1) \gamma_{H}\right)+2 \gamma_{H}\right)
$$

so that $\partial e / \partial I_{G}>0$ if $\lambda>\bar{\lambda}$. We summarize these findings in the following Figure, which is the same as Figure 5 in the paper.

Similarly to Figure 2, Figure 5 has an area (top area where $\lambda>\max \left\{\bar{\lambda}, \lambda_{3}\left(\gamma_{G}\right)\right\}$ ) where the emission level increases in the investment level in green innovation $\left(\partial e / \partial I_{G}>0\right)$. As the 
investment level increases, the emission of Firm 2 is reduced (as more consumers buy the cleaner product), but the emission of Firm 1 increases more than the decrease in the emission level of Firm 2.

\section{Profit-Maximizing Investment}

At the outset, Firm 1 will choose $I_{G}$ that solves the following problem:

$$
\left\{\begin{array}{c}
\operatorname{Max}\left\{\frac{1}{\lambda} \gamma_{H}\left(\gamma_{H}-\gamma_{G}\left(I_{G}\right)\right) \frac{\left(2 \lambda(v-c)-\lambda \gamma_{G}(v)(G+\tau+1)+\gamma_{G}\left(I_{G}\right)\right)^{2}}{\gamma_{G}\left(I_{G}\right)\left(4 \gamma_{H}-\gamma_{G}\left(I_{G}\right)\right)^{2}}-I_{G}\right\} \\
\text { s.t. } \gamma\left(I_{G}\right) \leq \gamma_{P} \equiv \gamma\left(I_{P G}\right)
\end{array}\right.
$$

Let $I^{D}(\tau)$ denote the solution of this maximization problem, which is either $I_{P G}$ (the minimum investment needed to obtain a patent) or $I_{G}^{d}$ (if it is larger than $I_{P G}$ ). Note that $I_{G}^{d}$ is the unconstrained profit-maximizing investment level, which satisfies the following first order condition:

$$
-\gamma_{G}^{\prime}(.) \gamma_{H} \frac{-\lambda\left((v-c)\left(4 \gamma_{G}^{2}-6 \gamma_{G} \gamma_{H}+8 \gamma_{H}^{2}\right)+\gamma_{G} \gamma_{H}(1+\tau+G)\left(4 \gamma_{H}-7 \gamma_{G}\right)\right)+\gamma_{G} \gamma_{H}\left(4 \gamma_{H}-7 \gamma_{G}\right)}{\lambda \gamma_{G}^{2}\left(4 \gamma_{H}-\gamma_{G}\right)^{3}}\left(\lambda\left(\gamma_{G}(G+\tau+1)-2(v-c)\right)-\gamma_{G}\right)=1
$$

Let $F\left(I_{G}^{d}, \tau\right)$ represent this first order condition, (26). The solution $I_{G}^{d}$ is a function of $\tau$ and, thus, by totally differentiating $F($.$) and rearranging the terms, we can obtain the sign of \partial I_{G}^{d} / \partial \tau$, which is the same as the sign of $\partial F / \partial \tau$, when the SOC condition is satisfied. We, thus, calculate the following:

$$
\frac{\partial F}{\partial \tau}=\frac{2\left(\gamma_{H}\left(4 \gamma_{H}-7 \gamma_{G}\right)-\lambda\left(\gamma_{H}\left(4 \gamma_{H}-7 \gamma_{G}\right)(G+\tau+1)+2(v-c)\left(\gamma_{G}+2 \gamma_{H}\right)\right)\right)}{\left(4 \gamma_{H}-\gamma_{G}\right)^{3}}
$$

which is negative if $\lambda>\widetilde{\lambda}$ where

$$
\widetilde{\lambda} \equiv \frac{\gamma_{H}\left(4 \gamma_{H}-7 \gamma_{G}\right)}{\gamma_{H}\left(4 \gamma_{H}-7 \gamma_{G}\right)(\underline{G}+\tau+1)+2(v-c)\left(\gamma_{G}+2 \gamma_{H}\right)} .
$$

Therefore, if the fraction of green conscious consumers is large enough, $\lambda>\widetilde{\lambda}$, as the tax increases the equilibrium investment level decreases, $\partial I_{G}^{d} / \partial \tau<0$.

\section{Emission levels in equilibrium}

First, in the case of Bertrand competition (if Firm 1 does not innovate, and both firms produce the dirty product), we obtain the same equilibrium as in the monopoly case: the emission level 
is given by $e^{c}=\lambda\left(v-c-\gamma_{H}(\underline{G}+\tau)\right)+\gamma_{H}(1-\lambda)$, which is decreasing with $\tau$. We define $\widehat{\tau}$ to be the value of the tax for which $e^{c}=0$,

$$
\widehat{\tau}=\frac{v-c}{\gamma_{H}}-\underline{G}+\frac{1-\lambda}{\lambda} .
$$

Then, we consider the case when the patenting constraint binds and Firm 1 must invest $I_{P G}\left(>I_{G}^{d}\right)$ in order to patent its innovation. In this case, the emission levels are, thus, given by $e_{G}\left(I_{P G}\right)=\gamma_{P} D_{G}(\tau)$ and $e_{H}\left(I_{P G}\right)=\gamma_{H} D_{H}(\tau)$, and both of them are decreasing with the tax, $\tau$, as $\partial e_{G}(\tau) / \partial \tau=\gamma_{P} \partial D_{G} / \partial \tau<0$ and $\partial e_{H} / \partial \tau=\gamma_{H} \partial D_{H} / \partial \tau<0$. Thus, the total emission level is given by

$$
e_{P G}=\frac{\gamma_{H}}{4 \gamma_{H}-\gamma_{P}}\left(3 \lambda(v-c)-\lambda(\underline{G}+\tau+1)\left(\gamma_{P}+2 \gamma_{H}\right)+2 \gamma_{H}+\gamma_{P}\right)
$$

which is decreasing with the tax as

$$
\frac{\partial e_{P G}}{\partial \tau}=-\lambda \gamma_{H} \frac{2 \gamma_{H}+\gamma_{P}}{4 \gamma_{H}-\gamma_{P}}<0
$$

We show that $e_{P G}$ intersects $e^{c}$ at

$$
\tau_{3}=\frac{v-c}{2 \gamma_{H}}-\underline{G}+\frac{1-\lambda}{\lambda}
$$

where $\tau_{3}<\widehat{\tau}$.

Evaluated at $\tau=0$, the total emission level is $e_{P G}(\tau=0)=\gamma_{P} D_{G}(0)+\gamma_{H} D_{H}(0)$, which is smaller than $e^{c}(\tau=0)$ if $\lambda(v-c)-2 \gamma_{H} \lambda(\underline{G}+1)+2 \gamma_{H}>0$.

When the patenting constraint does not bind, Firm 1 chooses $I_{G}^{*}$ that satisfies (26). In that case, the equilibrium emission level as a function of $\tau$ is given by:

$$
e(\tau)=e_{H}(\tau)+e_{G}(\tau)=\gamma_{H} D_{H}\left(I_{G}^{d}(\tau), \tau\right)+\gamma_{G}\left(I_{G}^{d}(\tau)\right) D_{G}\left(I_{G}^{d}(\tau), \tau\right) .
$$

Note that the demands are defined by (25) and (24) when Firm 1 chooses the profit-maximizing level of investment $I_{G}^{d}$, which means that $I_{G}^{d}$ is higher than the minimum requirement to obtain a patent $I_{P G}$.

First, we consider the impact of $\tau$ on the emission level of Firm $2, e_{H}(\tau)$,

$$
\frac{\partial e_{H}}{\partial \tau}=\gamma_{H}\left(\frac{\partial D_{H}}{\partial I_{G}} \frac{\partial I_{G}^{d}}{\partial \tau}+\frac{\partial D_{H}}{\partial \tau}\right)
$$


where $\partial D_{H} / \partial \tau<0$ and $\partial D_{H} / \partial I_{G}<0$. Thus, if $\partial I_{G}^{d} / \partial \tau>0$, which occurs when the fraction of green conscious consumers is not large, $\lambda<\widetilde{\lambda}$, then an increase in the tax reduces the emission level of Firm 2, $\partial e_{H} / \partial \tau<0$. However, if the fraction of green conscious consumers is large enough, $\lambda>\widetilde{\lambda}$, we have $\partial I_{G}^{d} / \partial \tau<0$, which implies that the impact of an increase of the tax on the emission level is ambiguous. If we calculate $\frac{\partial D_{H}}{\partial I_{G}} \frac{\partial I_{G}^{d}}{\partial \tau}+\frac{\partial D_{H}}{\partial \tau}$, we obtain the following:

$$
2 \gamma_{G}^{\prime}\left(I_{G}\right) I_{G}^{d^{\prime}}(\tau) \frac{\lambda(v-c)-2 \gamma_{H} \lambda(\underline{G}+\tau+1)+2 \gamma_{H}}{\left(4 \gamma_{H}-\gamma_{G}\right)^{2}}-2 \lambda \gamma_{H} \frac{\left(4 \gamma_{H}-\gamma_{G}\right)}{\left(4 \gamma_{H}-\gamma_{G}\right)^{2}},
$$

which is negative if

$$
\lambda<\frac{2 \gamma_{G}^{\prime}\left(I_{G}\right) I_{G}^{d^{\prime}}(\tau) \gamma_{H}}{-\left(\gamma_{G}^{\prime}\left(I_{G}\right) I_{G}^{d^{\prime}}(\tau)\left(v-c-2 \gamma_{H}(\underline{G}+\tau+1)\right)-\gamma_{H}\left(4 \gamma_{H}-\gamma_{G}\right)\right)},
$$

which is higher than 1 for $\gamma_{G}^{\prime}\left(I_{G}\right) I_{G}^{d^{\prime}}(\tau)\left(v-c-2 \gamma_{H}(\underline{G}+\tau)\right)>\gamma_{H}\left(4 \gamma_{H}-\gamma_{G}\right)$.

The effect of an increase of the tax on the emission level of Firm $1, e_{G}(\tau)=\gamma_{G}\left(I_{G}^{d}(\tau)\right) D_{G}\left(I_{G}^{d}(\tau), \tau\right)$, is determined by

$$
\begin{aligned}
& \frac{\partial e_{G}(\tau)}{\partial \tau}=\frac{\partial \gamma_{G}}{\partial I_{G}} \frac{\partial I_{G}^{d}}{\partial \tau} D_{G}(.)+\gamma_{G}\left(I_{G}^{*}\right)\left(\frac{\partial D_{G}}{\partial I_{G}} \frac{\partial I_{G}^{d}}{\partial \tau}+\frac{\partial D_{G}}{\partial \tau}\right),
\end{aligned}
$$

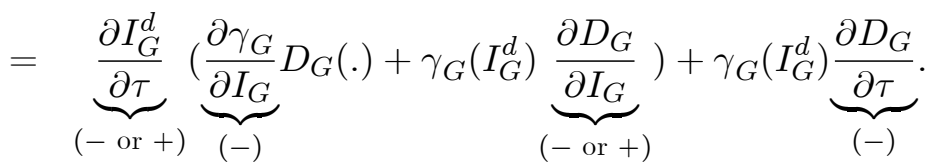

Thus, as $\partial D_{G} / \partial \tau<0$, if $\partial D_{G} / \partial I_{G}<0$ (if $\lambda<\lambda_{5}\left(\gamma_{G}\right)$ ) and $\partial I_{G}^{d} / \partial \tau>0$ (for $\lambda<\widetilde{\lambda}$ ) then $\partial e_{G}(\tau) / \partial \tau<0$. We also calculate that $\tilde{\lambda}>\lambda_{5}\left(\gamma_{G}\right)$ if $\left(4 \gamma_{H}-\gamma_{G}\right)\left(\gamma_{G}^{2}+4 \gamma_{H}\left(\gamma_{H}-2 \gamma_{G}\right)\right)>0$, or if $\gamma_{H}>2 \gamma_{G}$. Therefore, as long as $\gamma_{H}>2 \gamma_{G}$ the total emission level is decreasing with the tax, $\partial e(\tau) / \partial \tau<0$. Evaluated at $\tau=0, e(I)<e^{c}$, so that the emission level in the case of a duopoly is similar to the monopoly case as represented in Figures 3 and 4. 